QUARTERLY OF APPLIED MATHEMATICS

VOLUME LXVII, NUMBER 3

SEPTEMBER 2009, PAGES 503-540

S 0033-569X(09)01146-2

Article electronically published on May 6, 2009

\title{
EXISTENCE AND REGULARITY OF PULLBACK ATTRACTORS FOR AN INCOMPRESSIBLE NON-NEWTONIAN FLUID WITH DELAYS
}

\author{
BY
}

\begin{abstract}
CAIDI ZHAO (Department of Mathematics, Wenzhou University, Wenzhou 325035, People's Republic of China),
\end{abstract}

SHENGFAN ZHOU (Department of Applied Mathematics, Shanghai Normal University, Shanghai 200234, People's Republic of China),

AND

YONGSHENG LI (Department of Applied Mathematics, South China University of Technology, Guangdong, 510640, People's Republic of China)

Abstract. This paper studies an incompressible non-Newtonian fluid with delays in two-dimensional bounded domains. We first prove the existence and uniqueness of solutions. Then we establish the existence of pullback attractors $\left\{\mathscr{A}_{\mathcal{C}_{H}}(t)\right\}_{t \in \mathbb{R}}$ (has $L^{2}$-regularity), $\left\{\mathscr{A}_{\mathcal{C}_{W}}(t)\right\}_{t \in \mathbb{R}}$ (has $H^{2}$-regularity), and $\left\{\mathscr{A}_{E_{H}^{2}}(t)\right\}_{t \in \mathbb{R}}$ (has $L^{2}$-regularity), $\left\{\mathscr{A}_{E^{2}}(t)\right\}_{t \in \mathbb{R}}$ (has $H^{2}$-regularity) corresponding to two different processes associated to the fluid, respectively. Meanwhile, we verify the regularity of the pullback attractors by proving

$$
\mathscr{A}_{\mathcal{C}_{H}}(t)=\mathscr{A}_{\mathcal{C}_{W}}(t), \quad \mathscr{A}_{E_{H}^{2}}(t)=\mathscr{A}_{E_{W}^{2}}(t), \quad \forall t \in \mathbb{R}
$$

and

$$
\mathscr{A}_{E_{H}^{2}}(t)=J\left(\mathscr{A}_{\mathcal{C}_{H}}(t)\right)=J\left(\mathscr{A}_{\mathcal{C}_{W}}(t)\right)=\mathscr{A}_{E_{W}^{2}}(t), \quad \forall t \in \mathbb{R}
$$

where $J$ is a linear operator. By the regularity we reveal the pullback asymptotic smoothing effect of the fluid in the sense that the solutions become eventually more regular than the initial data. This effect implies, in the case of delays, that the regularity of the fluid in its history state does not play an important role on the regularity of its eventual state. Finally, we give some remarks.

Received February 20, 2008.

2000 Mathematics Subject Classification. Primary 35B41, 35Q35, 76D03.

Key words and phrases. Pullback attractor, evolution equations, incompressible non-Newtonian fluid, pullback asymptotic smoothing effect.

The work is supported by the NSF of China under Grant Numbers 10826091, 10771139, 10471047, and 10771074, the NSF of Zhejiang Province under Grant Number Y6080077 and the Innovation Program of Shanghai Municipal Education Commission under Grant 08ZZ70.

E-mail address: zhaocaidi@yahoo.com.cn 
1. Introduction. In this paper, we prove the unique existence of solutions and the existence and regularity of pullback attractors for the following incompressible nonNewtonian fluid with delays:

$$
\begin{gathered}
\frac{\partial u}{\partial t}+(u \cdot \nabla) u+\nabla p-\nabla \cdot \tau(e(u))=g(x)+f\left(t, u_{t}\right), \quad x=\left(x_{1}, x_{2}\right) \in \Omega, \\
\nabla \cdot u=\frac{\partial u_{1}}{\partial x_{1}}+\frac{\partial u_{2}}{\partial x_{2}}=0,
\end{gathered}
$$

where $\Omega$ is a smooth bounded domain of $\mathbb{R}^{2}$, the unknown vector function $u=u(x, t)=$ $\left(u_{1}, u_{2}\right)$ denotes the velocity of the fluid, $g(x)=\left(g_{1}, g_{2}\right)$ is the external force function, the scalar function $p$ represents the pressure and $f\left(t, u_{t}\right)$ denotes the delay term with delay function $u_{t}$ (see notation in Section 2). Equations (1.1)-(1.2) describe the motion of an isothermal incompressible viscous fluid in which the forcing term contains some delay features. These situations may occur, for example, when we want to control the system via applying a force which considers not only the present state but also the history state of the system. In (1.1), $\tau(e(u))=\left(\tau_{i j}(e(u))\right)_{2 \times 2}$, which is usually called the extra stress tensor of the fluid, is a matrix of order $2 \times 2$ defined as

$$
\tau_{i j}(e(u))=2 \mu_{0}\left(\varepsilon+|e|^{2}\right)^{-\alpha / 2} e_{i j}-2 \mu_{1} \Delta e_{i j}, \quad i, j=1,2,
$$

where

$$
e_{i j}=e_{i j}(u)=\frac{1}{2}\left(\frac{\partial u_{i}}{\partial x_{j}}+\frac{\partial u_{j}}{\partial x_{i}}\right), \quad|e|^{2}=\sum_{i, j=1}^{2}\left|e_{i j}\right|^{2},
$$

and $\mu_{0}, \mu_{1}, \alpha, \varepsilon$ are parameters which generally depend on the temperature and pressure of the fluid. In this paper, we assume that $\mu_{0}, \mu_{1}, \varepsilon$ are positive constants and $0<\alpha<1$. The extra stress tensor (1.3) was first formulated by Ladyzhenskaya [36] in the context of a non-Newtonian fluid. There are many works concerning the unique existence, regularity and long-term behavior of solutions to the addressed non-Newtonian fluid or its associated versions (see e.g. [4, 6, 7, 8, 9, 27, 32, 36, 41, 42, 57, 58).

The long-term behavior of dynamical systems is an interesting and challenging problem because it can provide important information on the future evolution of the system. The attractor is an important concept in the study of the long-term behavior of dynamical systems. There are many works concerning this subject (3, 24, 29, 37, 47, 52, 53]). For physical reasons (e.g. biological motivations (e.g., 35]) like species' growth), retarded differential equations have become an important area of applied mathematics (see [28, 30, 54]). There are many papers on retarded ODEs, PDEs and functional differential equations (see e.g. [3, 44, 45, 46]). For instance, Navier-Stokes equations with delays were systematically studied in [17, 18, 19. Many important and general results on delay dynamical systems on $\mathcal{C}([-\nu, 0], X)$ ( $X$ is a Hilbert space or Banach space) were presented in [54. Functional differential equations with delays were systematically studied in 28, 30, 54.

Recently, a new type of attractor, called the pullback attractor, has drawn much attention from mathematicians and physicists (see e.g. [25, 26, 33, 34, 38, 39, 48, 50, 51]). Actually, the theory of pullback attractors has been shown to be very useful in the understanding of the dynamics of non-autonomous and random dynamical systems, 
including those with delays (see e.g. [1, 19, 21, 22, 23, 48, 50, 51]). There are several versions of the concept of pullback attractors; see [4] for details. One of the advantages of the theory of pullback attractors is that it allows one to handle more general nonautonomous terms and it works under random environments as well (see 13). In fact, the theory of pullback attractors has been widely used and there are many works concerning this subject; one can refer to [10, [13,-16], 20, 23, 33, 34, 38, 39, 48, 55, 59.

We would like to point out that Caraballo and Real [19] started an investigation involving Navier-Stokes models in which the forcing term contains delays. The idea of the current paper originates from [19]. Caraballo and Real [19] proved the unique existence of pullback attractors $\left\{\mathcal{A}_{\mathcal{C}_{H}}(t)\right\}_{t \in \mathbb{R}} \subset \mathcal{C}_{H}$ and $\left\{\mathcal{A}_{M_{H}^{2}}(t)\right\}_{t \in \mathbb{R}} \subset H \times L_{H}^{2}$ (see notation in Section 2), respectively, for two different processes $\{U(t, \tau)\}_{t \geq \tau}$ and $\{S(t, \tau)\}_{t \geq \tau}$ associated to the Navier-Stokes models with delays. The main technique for the investigation in 19] involves two mappings $\tilde{U}(\cdot, \cdot)$ and $j$ which give some connection between the processes $\{U(t, \tau)\}_{t \geq \tau}$ and $\{S(t, \tau)\}_{t \geq \tau}$. Moreover, an interesting relation between $\left\{\mathcal{A}_{\mathcal{C}_{H}}(t)\right\}_{t \in \mathbb{R}}$ and $\left\{\mathcal{A}_{M_{H}^{2}}(t)\right\}_{t \in \mathbb{R}}$ is given via the mapping $j$. These techniques and arguments will also be applied by us. As will be shown below, the works of Caraballo and Real [19] greatly influence the presentation of this paper.

In this paper, we first aim to establish the existence of pullback attractors $\left\{\mathscr{A}_{\mathcal{C}_{H}}(t)\right\}_{t \in \mathbb{R}}$ $\subset \mathcal{C}_{H},\left\{\mathscr{A}_{E_{H}^{2}}(t)\right\}_{t \in \mathbb{R}} \subset H \times L_{H}^{2}$, and $\left\{\mathscr{A}_{\mathcal{C}_{W}}(t)\right\}_{t \in \mathbb{R}} \subset \mathcal{C}_{W},\left\{\mathscr{A}_{E_{W}^{2}}(t)\right\}_{t \in \mathbb{R}} \subset W \times L_{W}^{2}$ for the corresponding processes defined on different state spaces. For the existence of $\left\{\mathscr{A}_{\mathcal{C}_{H}}(t)\right\}_{t \in \mathbb{R}}$ and $\left\{\mathscr{A}_{E_{H}^{2}}(t)\right\}_{t \in \mathbb{R}}$, we use the technique and argument of [19] with some slight differences on calculations. This result seems a trivial generalization of [19 to the non-Newtonian fluid, but it make sense because it is the base of the regularity of the pullback attractor and furthermore it will provide us the existence of a family of uniformly bounded pullback absorbing sets in the space $W \times L_{W}^{2}$. Indeed, we will prove, using the argument of energy equality (see e.g. Ball [5]) that the obtained uniformly bounded pullback absorbing set in $W \times L_{W}^{2}$ is in fact pre-compact in $W \times L_{W}^{2}$, and then we prove the existence of the pullback attractor $\left\{\mathscr{A}_{E_{W}^{2}}(t)\right\}_{t \in \mathbb{R}} \subset W \times L_{W}^{2}$. We would like to point out that it seems difficult to obtain the existence of the pullback attractor $\left\{\mathscr{A}_{\mathcal{C}_{W}}(t)\right\}_{t \in \mathbb{R}}$ in $\mathcal{C}_{W}$ via the energy method. The main reason is that $\mathcal{C}_{W}$ is not a Hilbert space. One can see Remark 4.1 for details.

The second purpose of the current paper is to prove the regularity of the pullback attractors. We first establish

$$
\mathscr{A}_{E_{H}^{2}}(t)=\mathscr{A}_{E_{W}^{2}}(t), \quad \forall t \in \mathbb{R} .
$$

The main step is to utilize the Uniform Gronwall Lemma to prove that the solutions of the addressed delay system with initial data in any bounded set of $H \times L_{H}^{2}$ will enter a bounded set of $W \times L_{W}^{2}$ after large enough time. Then we use (1.4) and the relation between $\mathscr{A}_{\mathcal{C}_{H}}(t)$ and $\mathscr{A}_{E_{H}^{2}}(t)$ to prove that $\left\{\mathscr{A}_{\mathcal{C}_{H}}(t)\right\}_{t \in \mathbb{R}}$ is a family of compact sets of $\mathcal{C}_{W}$. This fact allows us to establish the existence of the pullback attractor $\left\{\mathscr{A}_{\mathcal{C}_{W}}(t)\right\}_{t \in \mathbb{R}}$ in $\mathcal{C}_{W}$. Meanwhile, the following regularity,

$$
\mathscr{A}_{\mathcal{C}_{H}}(t)=\mathscr{A}_{\mathcal{C}_{W}}(t), \forall t \in \mathbb{R},
$$


follows from the uniqueness of the uniformly bounded pullback attractor.

By (1.4) and (1.5), we can claim two conclusions. The first one is that the pullback attractors associated to (1.1)-(1.2) do not depend on the energy space chosen for the mathematical studying; the second one is the pullback asymptotic smoothing effect of the fluid in the sense that the solutions become eventually more regular (possessing $\mathrm{H}^{2}$ regularity) than the initial data (possessing $L^{2}$-regularity), which implies, in the case of delays, that the regularity of the fluid in its history state does not play an important role on the regularity of its eventual state. Also by (1.4), (1.5) and the definition of the linear operator $J$ (see (3.38) ), we have

$$
\mathscr{A}_{E_{H}^{2}}(t)=J\left(\mathscr{A}_{\mathcal{C}_{H}}(t)\right)=J\left(\mathscr{A}_{\mathcal{C}_{W}}(t)\right)=\mathscr{A}_{E_{W}^{2}}(t), \quad \forall t \in \mathbb{R} .
$$

Compared with the work of [59, the new problem encountered in this paper is that $\mathcal{C}_{H}$ and $\mathcal{C}_{W}$ are neither Hilbert spaces nor uniform convex Banach spaces. In [59], the authors of the present paper proved the existence and regularity of the cocycle attractor for the non-Newtonian fluid without delays, by establishing that the associated cocycle satisfies the pullback condition (PC) (see Lemma 2.3 in [59]) and by using the regularity of solutions and embedding theorems. Since $\mathcal{C}_{H}$ and $\mathcal{C}_{W}$ are not uniform convex Banach spaces, the argument used in Section 3 of [59] seems difficult to apply. Also, the method of energy equality is not applicable because $\mathcal{C}_{H}$ and $\mathcal{C}_{W}$ are not Hilbert spaces. At the same time, if the spatial domain is unbounded, the embedding $W \hookrightarrow H$ is no longer compact and the arguments used both in Section 3 and Section 4 of [59] are inadequate. We note that the approach presented in this paper is still valid for the case when the spatial domain is unbounded; see Remark 6.2 for details.

The paper is organized as follows. In the next section, we first introduce some notation and prove the unique existence of solutions to our problem. Then we recall some definitions and already known results concerning pullback attractors. In Section 3, we prove the existence of pullback attractors $\left\{\mathscr{A}_{\mathcal{C}_{H}}(t)\right\}_{t \in \mathbb{R}} \subset \mathcal{C}_{H}$ and $\left\{\mathscr{A}_{E_{H}^{2}}(t)\right\}_{t \in \mathbb{R}} \subset H \times L_{H}^{2}$ for two different processes defined on $\mathcal{C}_{H}$ and $H \times L_{H}^{2}$, respectively. In Section 4, we establish the existence of a pullback attractor $\left\{\mathscr{A}_{E_{W}^{2}}(t)\right\}_{t \in \mathbb{R}} \subset W \times L_{W}^{2}$. In Section 5, we first prove $\mathscr{A}_{E_{H}^{2}}(t)=\mathscr{A}_{E_{W}^{2}}(t)$ for all $t \in \mathbb{R}$. Then we establish the existence of the pullback attractor $\left\{\mathscr{A}_{\mathcal{C}_{W}}(t)\right\}_{t \in \mathbb{R}}$ in $\mathcal{C}_{W}$ and show $\mathscr{A}_{\mathcal{C}_{H}}(t)=\mathscr{A}_{\mathcal{C}_{W}}(t)$ for all $t \in \mathbb{R}$. We end the paper with some remarks in Section 6.

2. Preliminaries. In this paper, we use the following notation:

$L^{p}(\Omega)=$ the $2 \mathrm{D}$ vector Lebesgue space with norm $\|\cdot\|_{L^{p}(\Omega)}$; in particular, $\|\cdot\|_{L^{2}(\Omega)}=\|\cdot\|$,

$H^{m}(\Omega)=$ the $2 \mathrm{D}$ vector Sobolev space $\left\{\phi=\left(\phi_{1}, \phi_{2}\right) \in L^{2}(\Omega), \nabla^{k} \phi \in L^{2}(\Omega), k \leq m\right\}$ with norm $\|\cdot\|_{H^{m}(\Omega)}($ see $[2])$,

$H_{0}^{1}(\Omega)=$ closure of $\left\{\phi: \phi=\left(\phi_{1}, \phi_{2}\right) \in \mathcal{C}_{0}^{\infty}(\Omega) \times \mathcal{C}_{0}^{\infty}(\Omega)\right\}$ in $H^{1}(\Omega)$,

$\mathcal{V}=\left\{\phi \in \mathcal{C}_{0}^{\infty}(\Omega) \times \mathcal{C}_{0}^{\infty}(\Omega): \phi=\left(\phi_{1}, \phi_{2}\right), \nabla \cdot \phi=0\right\}$,

$H=$ closure of $\mathcal{V}$ in $L^{2}(\Omega)$ with norm $\|\cdot\|, \quad H^{\prime}=$ dual space of $H$,

$W=$ closure of $\mathcal{V}$ in $H^{2}(\Omega)$ with norm $\|\cdot\|_{W}, W^{\prime}=$ dual space of $W$,

$(\cdot, \cdot)=$ the inner product in $H, \quad\langle\cdot, \cdot\rangle=$ the dual pairing between $W$ and $W^{\prime}$,

$\mathcal{C}_{H}=\mathcal{C}([-\nu, 0] ; H), \quad \mathcal{C}_{W}=\mathcal{C}([-\nu, 0] ; W) \quad(\nu$ is a fixed positive number $)$, 
$L_{H}^{2}=L^{2}(-\nu, 0 ; H), \quad L_{W}^{2}=L^{2}(-\nu, 0 ; W)$,

$E_{H}^{2}=H \times L_{H}^{2}, \quad E_{W}^{2}=W \times L_{W}^{2}$,

$(\cdot, \cdot)_{E_{H}^{2}}=$ the inner product in $E_{H}^{2}$ defined as

$$
\left(\left(u_{0}, \psi\right),\left(v_{0}, \varphi\right)\right)_{E_{H}^{2}}=\left(u_{0}, v_{0}\right)+\int_{-\nu}^{0} \psi(\theta) \varphi(\theta) d \theta, \quad\left(u_{0}, \psi\right),\left(v_{0}, \varphi\right) \in E_{H}^{2},
$$

$\|\cdot\|_{E_{H}^{2}}=$ the norm of $E_{H}^{2}$ induced by $(\cdot, \cdot)_{E_{H}^{2}}$,

$(\cdot, \cdot)_{E_{W}^{2}}^{2}=$ the inner product in $E_{W}^{2}$ defined as

$$
\begin{aligned}
& \left(\left(u_{0}, \psi\right),\left(v_{0}, \varphi\right)\right)_{E_{W}^{2}} \\
& \quad=\sum_{k=0}^{2}\left(\nabla^{k} u_{0}, \nabla^{k} v_{0}\right)+\sum_{k=0}^{2} \int_{-\nu}^{0} \nabla^{k} \psi(\theta) \nabla^{k} \varphi(\theta) d \theta, \quad\left(u_{0}, \psi\right),\left(v_{0}, \varphi\right) \in E_{W}^{2},
\end{aligned}
$$

$\|\cdot\|_{E_{W}^{2}}=$ the norm of $E_{W}^{2}$ induced by $(\cdot, \cdot)_{E_{W}^{2}}$,

$\mathbb{R}=$ the set of real numbers, $\mathbb{N}=$ the set of positive integers,

$\mathbb{R}_{\tau}=[\tau,+\infty), \quad \mathbb{R}_{+}=[0,+\infty)$,

$\operatorname{Dist}_{E}(X, Y)=$ the Hausdorff semidistance between $X \subset E$ and $Y \subset E$ defined by

$$
\operatorname{Dist}_{E}(X, Y)=\sup _{x \in X} \inf _{y \in Y}\|x-y\|_{E}
$$

"—" and " $\rightarrow$ " denote convergence in the strong topology and the weak topology, respectively,

" $\hookrightarrow$ " denotes embedding between spaces.

We next introduce some operators so that we can put our problem into an abstract form. Set

$$
a(u, v)=\sum_{i, j, k=1}^{2}\left(\frac{\partial e_{i j}(u)}{\partial x_{k}}, \frac{\partial e_{i j}(v)}{\partial x_{k}}\right)=\sum_{i, j, k=1}^{2} \int_{\Omega} \frac{\partial e_{i j}(u)}{\partial x_{k}} \frac{\partial e_{i j}(v)}{\partial x_{k}} d x, u, v \in W .
$$

Lemma 2.1 (Bloom and Hao [8]). There exist two positive constants $c_{1}$ and $c_{2}$ which depend only on $\Omega$ such that

$$
c_{1}\|u\|_{W}^{2} \leq a(u, u) \leq c_{2}\|u\|_{W}^{2}, \quad \forall u \in W .
$$

From the definition of $a(\cdot, \cdot)$ and Lemma 2.1 we see that $a(\cdot, \cdot)$ defines a positive definite symmetric bilinear form on $W$. As a consequence of the Lax-Milgram Lemma, we obtain an isometric operator $A \in \mathscr{L}\left(W, W^{\prime}\right)$, via $\langle A u, v\rangle=a(u, v), \forall u, v \in W$. Moreover, let $D(A)=\{u \in W: A u \in H\}$. Then $D(A)$ is a Hilbert space and $A$ is also an isometry map from $D(A)$ to $H$. Indeed, $A=P \Delta^{2}$, where $P$ is the Leray projector from $L^{2}(\Omega)$ to $H$. For any $u \in D(A)$, we have

$$
c_{1}\|u\|_{W} \leq\|A u\|
$$

because by Lemma 2.1 we get

$$
c_{1}\|u\|_{W}^{2} \leq a(u, u)=\langle A u, u\rangle=(A u, u) \leq\|A u\|\|u\|_{W} .
$$


We also define a continuous trilinear form on $H_{0}^{1}(\Omega) \times H_{0}^{1}(\Omega) \times H_{0}^{1}(\Omega)$ as follows:

$$
b(u, v, w)=\sum_{i, j=1}^{2} \int_{\Omega} u_{i} \frac{\partial v_{j}}{\partial x_{i}} w_{j} d x, u, v, w \in H_{0}^{1}(\Omega) .
$$

Since $W \subset H_{0}^{1}(\Omega), b(\cdot, \cdot, \cdot)$ is continuous on $W \times W \times W$ and one can check

$$
b(u, v, w)=-b(u, w, v), \quad b(u, v, v)=0, \quad \forall u, v, w \in W .
$$

Now for any $u \in W$,

$$
\langle B(u), w\rangle=b(u, u, w), \forall w \in W,
$$

defines a continuous functional $B(u)$ from $W \times W$ to $W^{\prime}$. Finally, for $u \in W$, we set

$$
\mu(u)=2 \mu_{0}\left(\varepsilon+|e(u)|^{2}\right)^{-\alpha / 2}
$$

and define $N(u)$ as

$$
\langle N(u), v\rangle=\sum_{i, j=1}^{2} \int_{\Omega} \mu(u) e_{i j}(u) e_{i j}(v) d x, \forall v \in W .
$$

Then the functional $N(u)$ is continuous from $W$ to $W^{\prime}$. When $u \in D(A), N(u)$ can be extended to $H$ via

$$
\langle N(u), v\rangle=(N(u), v)=-\int_{\Omega}\{\nabla \cdot[\mu(u) e(u)]\} \cdot v d x, \quad \forall v \in H .
$$

Given $T>\tau$ and $u:[\tau-\nu, T) \mapsto H$, for each $t \in[\tau, T)$, we denote by $u_{t}=u_{t}(\cdot)$ the function defined on $[-\nu, 0]$ according to the relation $u_{t}(\theta)=u(t+\theta), \theta \in[-\nu, 0]$. In order to properly state the problem, we need some suitable hypotheses on the term containing delays. Let $f(\cdot, \cdot): \mathbb{R} \times \mathcal{C}_{H} \longmapsto H$ satisfy the following assumptions:

$\left(\mathbf{A}_{\mathbf{1}}\right) \quad \forall u \in \mathcal{C}_{H}, \mathbb{R} \ni t \longmapsto f(t, u) \in H$ is measurable.

$\left(\mathbf{A}_{\mathbf{2}}\right) f(t, 0)=0, \quad \forall t \in \mathbb{R}$.

$\left(\mathbf{A}_{3}\right)$ There exists a positive constant $C_{f}$ such that $\forall t \in \mathbb{R}, \forall u, v \in \mathcal{C}_{H}$, we have

$$
\|f(t, u)-f(t, v)\| \leq C_{f}\|u-v\|_{\mathcal{C}_{H}} .
$$

$\left(\mathbf{A}_{4}\right)$ There exist two positive constants $\beta_{0}$ and $L_{f}$ such that for any $\beta \in\left[0, \beta_{0}\right)$, any $t>\tau$ and any $u, v \in \mathcal{C}([\tau-\nu, t] ; H)$, it follows that

$$
\int_{\tau}^{t} e^{\beta \xi}\left\|f\left(t, u_{\xi}\right)-f\left(t, v_{\xi}\right)\right\|^{2} d \xi \leq L_{f}^{2} \int_{\tau-\nu}^{t} e^{\beta \xi}\|u(\xi)-v(\xi)\|^{2} d \xi .
$$

$\left(\mathbf{A}_{\mathbf{5}}\right) L_{f}<c_{1} \mu_{1}$.

REMARK 2.1. Caraballo and Real [19] presented a specific function which satisfies all assumptions $\left(\mathbf{A}_{\mathbf{1}}\right)-\left(\mathbf{A}_{\mathbf{4}}\right)$; see pp. 292, 293 in [19] for details. If we let the constant $\mu_{1}$ be sufficiently large, then $\left(\mathbf{A}_{\mathbf{1}}\right)-\left(\mathbf{A}_{\mathbf{5}}\right)$ are satisfied.

Indeed, $\left(\mathbf{A}_{\mathbf{1}}\right)-\left(\mathbf{A}_{\mathbf{3}}\right)$ imply that for a given $u \in \mathcal{C}([\tau-\nu, T] ; H)$ the function $f\left(t, u_{t}\right)$ : $[\tau, T] \ni t \longmapsto H$ is measurable (see [19]) and belongs to $L^{\infty}(\tau, T ; H)$. Then $\left(\mathbf{A}_{\mathbf{4}}\right)$ implies that the mapping $\mathcal{F}: \mathcal{C}([\tau-\nu, T] ; H) \ni u \longmapsto f\left(t, u_{t}\right) \in L^{2}(\tau, T ; H)$ can be 
extended uniquely to a mapping which is uniformly continuous from $L^{2}(\tau-\nu, T ; H)$ into $L^{2}(\tau, T ; H)$. Thus, for any $u, v \in L^{2}(\tau, T ; H)$, it follows that

$$
\int_{\tau}^{t}\left\|f\left(\xi, u_{\xi}\right)-f\left(\xi, v_{\xi}\right)\right\|^{2} d \xi \leq L_{f}^{2} \int_{\tau-\nu}^{t}\|u(\xi)-v(\xi)\|^{2} d \xi
$$

By the above notation and analysis, we consider problem (1.1)-(1.3), excluding the pressure $p$, in the solenoidal vector field as

$$
\left\{\begin{array}{l}
\text { For each } \tau \in \mathbb{R} \text { and } \forall T>\tau, \text { find } \\
u \in L^{2}(\tau-\nu, T ; H) \cap L^{2}(\tau, T ; W) \cap L^{\infty}(\tau, T ; H), \\
\text { such that } \frac{\partial}{\partial t} u(t)+2 \mu_{1} A u(t)+B(u(t))+N(u(t))=g(x)+f\left(t, u_{t}\right) \\
\text { in } \mathcal{D}\left(\tau,+\infty ; W^{\prime}\right), u(\tau)=u_{0}, \quad u(t)=\psi(t-\tau), \quad t \in[\tau-\nu, \tau) .
\end{array}\right.
$$

For problem (2.14), we have the following existence and uniqueness result.

Theorem 2.1. Assume $\left(\mathbf{A}_{\mathbf{1}}\right)-\left(\mathbf{A}_{\mathbf{5}}\right)$ hold and $g \in H$. Then for each $\tau \in \mathbb{R}$, it follows that

(1) For any $\left(u_{0}, \psi\right) \in E_{H}^{2}$, there exists a unique solution u to problem (2.14); moreover, $u \in \mathcal{C}([\tau,+\infty) ; H)$.

(2) For any $\left(u_{0}, \psi\right) \in E_{W}^{2}$, problem (2.14) admits a unique solution u satisfying

$$
u \in L^{2}(\tau-\nu, T ; H) \cap L^{2}(\tau, T ; D(A)) \cap \mathcal{C}([\tau, T] ; W) \text { and } \frac{\partial u}{\partial t} \in L^{2}(\tau, T ; H) .
$$

In particular, if $\psi \in \mathcal{C}_{W}$ and $u_{0}=\psi(0)$, then $u \in \mathcal{C}([\tau-\nu,+\infty) ; W)$.

Proof. For the existence of solutions, we can use the Galerkin approximation scheme similar to that in Constantin and Foias [12, and Caraballo and Real [19]. Here we only emphasize the different places involving the new term $N(\cdot)$ and the higher-order term $A(\cdot)$. Indeed, by the definition of $A$ and the classical spectral theory of elliptic operators (see e.g. 41]), we see that the operator $A$ possesses a family of eigenfunctions $\left\{w_{n}\right\}_{n=1}^{\infty} \subset W \cap D(A)$, which form a basis of $W$ and are orthonormal in $H$. We consider the subspace $W_{m}$ of $W$ spanned by $w_{1}, w_{2}, \ldots, w_{m}$, and the projector $P_{m}: H \longmapsto W_{m}$ defined as

$$
P_{m} u=\sum_{i=1}^{m}\left(u, w_{i}\right) w_{i}, \quad u \in H .
$$

Define

$$
u^{m}(t)=\sum_{i=1}^{m} \gamma_{m i}(t) w_{i}
$$

and consider the following problem:

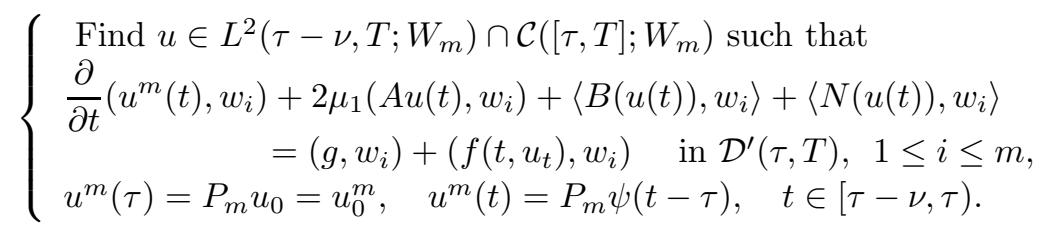

Note that problem (2.16) is a system of ordinary differential equations with respect to the unknown functions $\gamma^{m}(t)=\left(\gamma_{m 1}(t), \ldots, \gamma_{m m}(t)\right)$. We can obtain the existence of solutions to problem (2.16) defined on an interval $\left[\tau, t_{*}\right]\left(\tau<t_{*} \leq T\right)$ by applying 
essentially the same proof as that in Caraballo and Real [17. We next derive the a priori estimates which show that one can take $t_{*}=T$. Indeed, multiplying the equation in (2.16) by $\gamma_{m i}(t)$ and summing in $i$, we obtain by using Lemma 2.1 that for all $t \in\left[\tau, t_{*}\right]$,

$$
\begin{aligned}
& \frac{1}{2} \frac{d}{d t}\left\|u^{m}(t)\right\|^{2}+2 c_{1} \mu_{1}\left\|u^{m}(t)\right\|_{W}^{2}+\left\langle B\left(u^{m}(t)\right), u^{m}(t)\right\rangle+\left\langle N\left(u^{m}(t)\right), u^{m}(t)\right\rangle \\
\leq & \frac{1}{2} \frac{d}{d t}\left\|u^{m}(t)\right\|^{2}+2 \mu_{1} a\left(u^{m}(t), u^{m}(t)\right)+\left\langle B\left(u^{m}(t)\right), u^{m}(t)\right\rangle+\left\langle N\left(u^{m}(t)\right), u^{m}(t)\right\rangle \\
= & \left(g, u^{m}(t)\right)+\left(f\left(t, u_{t}^{m}\right), u^{m}(t)\right),
\end{aligned}
$$

integrating which over $[\tau, t]$, we have

$$
\begin{aligned}
& \frac{1}{2}\left\|u^{m}(t)\right\|^{2}+2 c_{1} \mu_{1} \int_{\tau}^{t}\left\|u^{m}(\xi)\right\|_{W}^{2} d \xi \\
& +\int_{\tau}^{t}\left\langle B\left(u^{m}(\xi)\right), u^{m}(\xi)\right\rangle d \xi+\int_{\tau}^{t}\left\langle N\left(u^{m}(\xi)\right), u^{m}(\xi)\right\rangle d \xi \\
& \quad=\frac{1}{2}\left\|u_{0}^{m}\right\|^{2}+\int_{\tau}^{t}\left(g, u^{m}(\xi)\right) d \xi+\int_{\tau}^{t}\left(f\left(\xi, u_{\xi}^{m}\right), u^{m}(\xi)\right) d \xi
\end{aligned}
$$

Now by (2.9) and the definition of the operator $N(\cdot)$, we get

$$
\begin{aligned}
& \int_{\tau}^{t}\left\langle B\left(u^{m}(\xi)\right), u^{m}(\xi)\right\rangle d \xi=0 \\
& \int_{\tau}^{t}\left\langle N\left(u^{m}(\xi)\right), u^{m}(\xi)\right\rangle d \xi \geq 0 .
\end{aligned}
$$

Also, we have

$$
\int_{\tau}^{t}\left(g, u^{m}(\xi)\right) d \xi \leq \frac{1}{2 c_{1} \mu_{1}} \int_{\tau}^{t}\|g\|^{2} d \xi+\frac{c_{1} \mu_{1}}{2} \int_{\tau}^{t}\left\|u^{m}(\xi)\right\|_{W}^{2} d \xi,
$$

and by $\left(\mathbf{A}_{\mathbf{2}}\right),\left(\mathbf{\mathbf { A } _ { 4 }}\right)$ and $\left(\mathbf{A}_{\mathbf{5}}\right)$,

$$
\begin{aligned}
\int_{\tau}^{t}\left(f\left(\xi, u_{\xi}^{m}\right), u^{m}(\xi)\right) d \xi & =\int_{\tau}^{t}\left(f\left(\xi, u_{\xi}^{m}\right)-f(\xi, 0), u^{m}(\xi)\right) d \xi \\
& \leq \int_{\tau}^{t}\left\|f\left(\xi, u_{\xi}^{m}\right)-f(\xi, 0)\right\|\left\|u^{m}(\xi)\right\| d \xi \\
& \leq \frac{1}{2 c_{1} \mu_{1}} \int_{\tau}^{t}\left\|f\left(\xi, u_{\xi}^{m}\right)-f(\xi, 0)\right\|^{2} d \xi \\
& \leq \frac{L_{f}^{2}}{2 c_{1} \mu_{1}} \int_{\tau-\nu}^{\tau}\left\|u^{m}(\xi)\right\|^{2} d \xi+\frac{L_{f}^{2}+c_{1}^{2} \mu_{1}^{2}}{2 c_{1} \mu_{1}} \int_{\tau}^{t}\left\|u^{m}(\xi)\right\|_{W}^{2} d \xi \\
& =\frac{L_{f}^{2}}{2 c_{1} \mu_{1}}\left\|P_{m} \psi(\xi)\right\|_{L_{H}^{2}}^{2}+\frac{L_{f}^{2}+c_{1}^{2} \mu_{1}^{2}}{2 c_{1} \mu_{1}} \int_{\tau}^{t}\left\|u^{m}(\xi)\right\|_{W}^{2} d \xi \\
& \leq \frac{L_{f}^{2}}{2 c_{1} \mu_{1}}\|\psi\|_{L_{H}^{2}}^{2}+c_{1} \mu_{1} \int_{\tau}^{t}\left\|u^{m}(\xi)\right\|_{W}^{2} d \xi
\end{aligned}
$$


It then follows from (2.17)-(2.21) that

$$
\left\|u^{m}(t)\right\|^{2}+c_{1} \mu_{1} \int_{\tau}^{t}\left\|u^{m}(\xi)\right\|_{W}^{2} d \xi \leq\left\|u_{0}\right\|^{2}+\frac{1}{c_{1} \mu_{1}} \int_{\tau}^{t}\|g\|^{2} d \xi+\frac{L_{f}^{2}}{c_{1} \mu_{1}}\|\psi\|_{L_{H}^{2}}
$$

by which we can take constants (depending on $u_{0}, c_{1}, L_{f}, \psi, g, T$, but not on $m$ and $t_{*}$ ) $M_{1}$ and $M_{2}$ such that

$$
\sup _{t \in\left[\tau, t_{*}\right]}\left\|u^{m}(t)\right\|^{2} \leq M_{1}, \quad \int_{\tau}^{t_{*}}\left\|u^{m}(\xi)\right\|_{W}^{2} d \xi \leq M_{2} .
$$

Thus, we can take $t_{*}=T$ and conclude that the sequence $\left\{u^{m}\right\}_{m=1}^{\infty}$ is bounded in $L^{2}(\tau, T ; W) \cap L^{\infty}(\tau, T ; H)$. Note that $u^{m}(t)=P_{m} \psi(t-\tau)$ for $t \in[\tau-\nu, \tau)$ and by the choice of the basis $\left\{w_{i}\right\}_{i=1}^{\infty}$, we find that for $t \in[\tau-\nu, \tau)$ the sequence $\left\{u^{m}\right\}_{m=1}^{\infty}$ converges to $\psi$ in $L_{W}^{2}$. Consequently, $f\left(\cdot, u^{m}\right)$ is bounded in $L^{2}\left(\tau, T ; W^{\prime}\right)$, and by Lemma 2.2 of [58, $B\left(u^{m}\right)$ and $N\left(u^{m}\right)$ are bounded in $L^{2}\left(\tau, T ; W^{\prime}\right)$. Then using the same reasoning as in [58] we can prove that the sequence $\left\{\frac{\partial u^{m}}{\partial t}\right\}_{m=1}^{\infty}$ is bounded in $L^{2}\left(\tau, T ; W^{\prime}\right)$. By Lemma 2.2 below and the assumptions on $f$, we see that there exist a subsequence (extract if necessary) of $\left\{u^{m}\right\}_{m=1}^{\infty}$ and $u \in L^{2}(\tau-\nu, T ; W)$ such that

$$
\left\{\begin{array}{l}
u^{m} \longrightarrow u \text { weakly in } L^{2}(\tau-\nu, T ; W), \\
u^{m} \longrightarrow u \text { strongly in } L^{2}(\tau, T ; H), \\
u^{m} \longrightarrow u \text { weakly-star in } L^{\infty}(\tau-\nu, T ; H), \\
f\left(\cdot, u^{m}\right) \longrightarrow f(\cdot, u) \text { weakly in } L^{2}\left(\tau-\nu, T ; W^{\prime}\right) .
\end{array}\right.
$$

By (2.22), we can take limits (similar to that of Bloom and Hao 8]) in (2.16) after integrating over $(\tau, t)$ (for $t \in(\tau, T)$ ), and get that $u$ is the solution to problem (2.14). The existence of solutions has been proved. The uniqueness of solutions will follow directly from Lemma 3.1 in Section 3. We end the proof of (1). The proof of (2) is similar to that of (1) and we omit it here. This concludes the proof of Theorem 2.1.

We next recall some definitions and results concerning the pullback attractors. These definitions and results can be found in [19, 24].

Let $(E, d)$ be a complete metric space and $\mathcal{B}(E)$ the collection of all bounded sets of $E$.

Definition 2.1. A two-parameter mapping $\{V(t, \tau)\}_{t \geq \tau}$ is said to be a process on $E$ if

(i) $V(t, \tau): E \mapsto E$ for all $t \geq \tau, \tau \in \mathbb{R}$;

(ii) $V(\tau, \tau)=$ Id (identity operator in $E$ ), $\tau \in \mathbb{R}$;

(iii) $V(t, s) V(s, \tau)=V(t, \tau)$ for all $t \geq s \geq \tau$.

If, in addition,

(iv) $V(t, \tau) x$ is jointly continuous in $(t, \tau, x)$ for all $t \geq \tau, \tau \in \mathbb{R}, x \in E$, then $\{V(t, \tau)\}_{t \geq \tau}$ is said to be a continuous process on $E$.

Definition 2.2. Let $\{V(t, \tau)\}_{t \geq \tau}$ be a process on $E$. A family of sets $\{\mathcal{B}(t)\}_{t \in \mathbb{R}} \subset E$ is said to be pullback absorbing for the process $V$ if for any $t \in \mathbb{R}$ and any $\mathcal{B} \in \mathcal{B}(E)$, there exists $T_{\mathcal{B}}(t)>0$ such that $V(t, t-s) \mathcal{B} \subset \mathcal{B}(t), \forall s \geq T_{\mathcal{B}}(t)$. The absorption is said to be uniform if $T_{\mathcal{B}}(t)$ does not depend on $t$.

Definition 2.3. Let $\{V(t, \tau)\}_{t \geq \tau}$ be a process on $E$. A family of compact sets $\{\mathcal{A}(t)\}_{t \in \mathbb{R}}$ is said to be a (global) pullback attractor for $\{V(t, \tau)\}_{t \geq \tau}$ if it satisfies 
(i) Invariance: $V(t, \tau) \mathcal{A}(\tau)=\mathcal{A}(t), \quad \forall t \geq \tau$, and

(ii) Pullback attracting: $\lim _{s \rightarrow+\infty} \operatorname{Dist}_{E}(V(t, t-s) \mathcal{B}, \mathcal{A}(t))=0, \forall \mathcal{B} \in \mathcal{B}(E), \forall t \in \mathbb{R}$.

REMark 2.2. The pullback attractor given by Definition 2.2 is indeed global. For other versions of the definition of pullback attractor, e.g., global pullback $\mathscr{D}$-attractor, one can refer to [14, 19, 51]. Also, the above defined global pullback attractor possesses the weak attracting property of kernel sections; see 24] for details.

For the existence of pullback attractors, the following result was proved in [11, 51].

TheOREM $2.2([51])$. Let $\{V(t, \tau)\}_{t>\tau}$ be a continuous process on $E$. If there exists a family of compact pullback absorbing sets $\{\mathcal{B}(t)\}_{t \in \mathbb{R}} \subset E$, then there exists a pullback attractor $\{\mathcal{A}(t)\}_{t \in \mathbb{R}}$, and $\mathcal{A}(t) \subset \mathcal{B}(t)$ for all $t \in \mathbb{R}$. Moreover

$$
\mathcal{A}(t)=\overline{\bigcup_{\mathcal{B} \in \mathcal{B}(E)} \omega_{\mathcal{B}}(t)}, \quad \text { where } \quad \omega_{\mathcal{B}}(t)=\bigcap_{n \in \mathbb{N}} \overline{\bigcup_{s \geq n} V(t, t-s) \mathcal{B}} .
$$

REMARK 2.3. Caraballo and Real [19] pointed out that if the family of sets $\{\mathcal{A}(t)\}_{t \in \mathbb{R}}$ is uniformly (with respect to $t \in \mathbb{R}$ ) bounded or the family of compact absorbing sets is uniformly bounded, then the pullback attractor is unique. But the uniqueness of the pullback attractor defined above does not hold in general.

We conclude this section with the following results.

Lemma 2.2 (24). Let $E_{0}, E_{1}, E$ be three Banach spaces satisfying $E_{1} \hookrightarrow E \subset E_{0}$, where the embedding $E_{1} \hookrightarrow E$ is compact. Assume $p_{1} \geq 1$ and $p_{0}>1$. Set

$$
W_{p_{1}, p_{0}}\left(0, T ; E_{1}, E_{0}\right)=\left\{\psi(t), t \in[0, T]: \psi(t) \in L^{p_{1}}\left(0, T ; E_{1}\right), \psi^{\prime}(t) \in L^{p_{0}}\left(0, T ; E_{0}\right)\right\} .
$$

Then the embedding is compact: $W_{p_{1}, p_{0}}\left(0, T ; E_{1}, E_{0}\right) \hookrightarrow L^{p_{1}}(0, T ; E), \forall T>0$.

Lemma 2.3 (Uniform Gronwall Lemma [53]). Let $\Upsilon(t), \Phi(t), \Psi(t)$ be three positive locally integrable functions on $[\tau,+\infty)$ such that $\Phi^{\prime}$ is locally integrable on $[\tau,+\infty)$ and

$$
\begin{gathered}
\frac{d \Phi(t)}{d t} \leq \Upsilon(t) \Phi(t)+\Psi(t) \quad \text { for } \quad t \geq \tau, \\
\int_{t}^{t+r} \Upsilon(s) d s \leq a_{1}, \quad \int_{t}^{t+r} \Psi(s) d s \leq a_{2}, \quad \int_{t}^{t+r} \Phi(s) d s \leq a_{3}, \quad \text { for } t \geq \tau,
\end{gathered}
$$

where $r, a_{1}, a_{2}$ and $a_{3}$ are positive constants. Then

$$
\Phi(t+r) \leq\left(\frac{a_{3}}{r}+a_{2}\right) e^{a_{1}}, \quad \forall t \geq \tau .
$$

3. Existence of pullback attractors with $L^{2}$-regularity. In this section, we will prove the existence of pullback attractors $\left\{\mathscr{A}_{\mathcal{C}_{H}}(t)\right\}_{t \in \mathbb{R}}$ and $\left\{\mathscr{A}_{E_{H}^{2}}(t)\right\}_{t \in \mathbb{R}}$ in the spaces $\mathcal{C}_{H}$ and $E_{H}^{2}$, respectively. We see that both $\mathcal{C}_{H}$ and $E_{H}^{2}$ possess $L^{2}$-regularity. As mentioned previously in the introduction, the idea and arguments of this section originate from Caraballo and Real [19].

Lemma 3.1. Assume $\left(\mathbf{A}_{\mathbf{1}}\right)-\left(\mathbf{A}_{\mathbf{5}}\right)$ hold and $g \in H$. Then for any given initial time $\tau \in \mathbb{R}$, the following hold. 
(I) Let $\left(u_{0}, \psi\right),\left(v_{0}, \varphi\right) \in E_{H}^{2}$ be two couples of initial data, and let $u(\cdot)=u\left(\cdot ; \tau,\left(u_{0}, \psi\right)\right)$ and $v(\cdot)=v\left(\cdot ; \tau,\left(v_{0}, \varphi\right)\right)$ be the corresponding solutions to (2.14). Then there exists a constant $c>0$ (independent of the initial time and data) such that

$$
\begin{aligned}
\|u(t)-v(t)\|^{2} \leq\left(\frac{L_{f}^{2}}{c_{1} \mu_{1}}\|\psi-\varphi\|_{L_{H}^{2}}^{2}+\left\|u_{0}-v_{0}\right\|^{2}\right) \\
\quad \cdot \exp \left(\int_{\tau}^{t}\left(\frac{c^{2}}{2 c_{1} \mu_{1}}\|u(\xi)\|_{W}^{2}+\frac{L_{f}^{2}}{c_{1} \mu_{1}}\right) d \xi\right), \quad \forall t \geq \tau .
\end{aligned}
$$

(II) For $\forall t \geq \tau$, denote by $u_{t}(\cdot)=u_{t}\left(\cdot ; \tau,\left(u_{0}, \psi\right)\right)$ and $v_{t}(\cdot)=v_{t}\left(\cdot ; \tau,\left(v_{0}, \varphi\right)\right)$ the corresponding solutions to (2.14) with initial conditions $\left(u_{0}, \psi\right),\left(v_{0}, \varphi\right) \in E_{H}^{2}$. Then

$\left\|u_{t}-v_{t}\right\|_{L_{H}^{2}}^{2} \leq\left(\left(\frac{L_{f}^{2} \nu}{c_{1} \mu_{1}}+1\right)\|\psi-\varphi\|_{L_{H}^{2}}^{2}+\nu\left\|u_{0}-v_{0}\right\|^{2}\right) \exp \left(\int_{\tau}^{t} \frac{L_{f}^{2}+c}{c_{1} \mu_{1}}\|u(\xi)\|_{W}^{2} d \xi\right)$.

Moreover, if $t \geq \tau+\nu$, then

$\left\|u_{t}-v_{t}\right\|_{\mathcal{C}_{H}}^{2} \leq\left(\frac{L_{f}^{2}}{c_{1} \mu_{1}}\|\psi-\varphi\|_{L_{H}^{2}}^{2}+\left\|u_{0}-v_{0}\right\|^{2}\right) \exp \left(\int_{\tau}^{t}\left(\frac{c^{2}}{2 c_{1} \mu_{1}}\|u(\xi)\|_{W}^{2}+\frac{L_{f}^{2}}{c_{1} \mu_{1}}\right) d \xi\right)$.

(III) If $\psi, \varphi \in \mathcal{C}_{H}$ and $u_{0}=\psi(0), v_{0}=\varphi(0)$, then for any $t \geq \tau$,

$$
\|u(t)-v(t)\|_{\mathcal{C}_{H}}^{2} \leq\left(\frac{\nu L_{f}^{2}}{c_{1} \mu_{1}}+1\right)\|\psi-\varphi\|_{\mathcal{C}_{H}}^{2} \exp \left(\int_{\tau}^{t}\left(\frac{c^{2}}{2 c_{1} \mu_{1}}\|u(\xi)\|_{W}^{2}+\frac{L_{f}^{2}}{c_{1} \mu_{1}}\right) d \xi\right) .
$$

Proof. (I) Set $w=u-v$. Then $w$ is a solution of the following problem:

$$
\begin{aligned}
& \frac{\partial w(t)}{\partial t}+2 \mu_{1} A w(t)+B(u(t))-B(v(t))+N(u(t))-N(v(t)) \\
&=f\left(t, u_{t}\right)-f\left(t, v_{t}\right), \quad t>\tau, \\
& w(\tau)=u_{0}-v_{0}, \quad w(t)=\psi(t-\tau)-\varphi(t-\tau), \quad t \in[\tau-\nu, \tau) .
\end{aligned}
$$

Multiplying (3.23) by $w(t)$, we get

$$
\begin{aligned}
& \frac{1}{2} \frac{d}{d t}\|w(t)\|^{2}+2 \mu_{1} a(w(t), w(t))+\langle B(u(t))-B(v(t)), w\rangle \\
& \quad+\langle N(u(t))-N(v(t)), w(t)\rangle=\left(f\left(t, u_{t}\right)-f\left(t, v_{t}\right), w(t)\right), \quad t>\tau .
\end{aligned}
$$

Integrating by parts, we have

$$
\langle B(u)-B(v), w\rangle=b(u, u, w)-b(v, v, w)=b(w, u, w) .
$$

Then using the Hölder and Gagliardo-Nirenberg inequalities, we get

$$
|\langle B(u)-B(v), w\rangle|=|b(w, u, w)| \leq c_{1} \mu_{1}\|w\|_{W}^{2}+\frac{c^{2}}{4 c_{1} \mu_{1}}\|w\|^{2}\|u\|_{W}^{2} ;
$$

hereafter $c$ is a positive constant depending on the Gagliardo-Nirenberg inequality and thus essentially on $\Omega$. Since $\alpha \in(0,1)$, we have (see [41])

$$
\langle N(u)-N(v), w\rangle=\sum_{i, j=1}^{2} \int_{\Omega}\left[\mu(u) e_{i j}(u)-\mu(v) e_{i j}(v)\right] e_{i j}(w) d x \geq 0 .
$$


Also, clearly,

$$
\left(f\left(t, u_{t}\right)-f\left(t, v_{t}\right), w(t)\right) \leq \frac{1}{2 c_{1} \mu_{1}}\left\|f\left(t, u_{t}\right)-f\left(t, v_{t}\right)\right\|^{2}+\frac{c_{1} \mu_{1}}{2}\|w(t)\|_{W}^{2} .
$$

It then follows from (3.25)-(3.29) that

$$
\frac{d}{d t}\|w(t)\|^{2}+c_{1} \mu_{1}\|w(t)\|_{W}^{2} \leq \frac{c^{2}}{2 c_{1} \mu_{1}}\|w(t)\|^{2}\|u(t)\|_{W}^{2}+\frac{1}{c_{1} \mu_{1}}\left\|f\left(t, u_{t}\right)-f\left(t, v_{t}\right)\right\|^{2}
$$

and thus

$$
\frac{d}{d t}\|w(t)\|^{2} \leq \frac{c^{2}}{2 c_{1} \mu_{1}}\|w(t)\|^{2}\|u(t)\|_{W}^{2}+\frac{1}{c_{1} \mu_{1}}\left\|f\left(t, u_{t}\right)-f\left(t, v_{t}\right)\right\|^{2} .
$$

Integrating (3.31) over $[\tau, t]$ and using (2.13), we obtain

$$
\begin{aligned}
& \|w(t)\|^{2}-\|w(\tau)\|^{2} \\
\leq & \frac{c^{2}}{2 c_{1} \mu_{1}} \int_{\tau}^{t}\|w(\xi)\|^{2}\|u(\xi)\|_{W}^{2} d \xi+\frac{1}{c_{1} \mu_{1}} \int_{\tau}^{t}\left\|f\left(\xi, u_{\xi}\right)-f\left(\xi, v_{\xi}\right)\right\|^{2} d \xi \\
\leq & \frac{c^{2}}{2 c_{1} \mu_{1}} \int_{\tau}^{t}\|w(\xi)\|^{2}\|u(\xi)\|_{W}^{2} d \xi+\frac{L_{f}^{2}}{c_{1} \mu_{1}} \int_{\tau-\nu}^{t}\|u(\xi)-v(\xi)\|^{2} d \xi \\
\leq & \frac{L_{f}^{2}}{c_{1} \mu_{1}}\|\psi-\varphi\|_{L_{H}^{2}}^{2}+\int_{\tau}^{t}\left(\frac{c^{2}}{2 c_{1} \mu_{1}}\|u(\xi)\|_{W}^{2}+\frac{L_{f}^{2}}{c_{1} \mu_{1}}\right)\|w(\xi)\|^{2} d \xi
\end{aligned}
$$

whence

$\|w(t)\|^{2} \leq\left\|u_{0}-v_{0}\right\|^{2}+\frac{L_{f}^{2}}{c_{1} \mu_{1}}\|\psi-\varphi\|_{L_{H}^{2}}^{2}+\int_{\tau}^{t}\left(\frac{c^{2}}{2 c_{1} \mu_{1}}\|u(\xi)\|_{W}^{2}+\frac{L_{f}^{2}}{c_{1} \mu_{1}}\right)\|w(\xi)\|^{2} d \xi, \forall t \geq \tau$,

and from which it follows by using the Gronwall Lemma that

$$
\|w(t)\|^{2} \leq\left(\left\|u_{0}-v_{0}\right\|^{2}+\frac{L_{f}^{2}}{c_{1} \mu_{1}}\|\psi-\varphi\|_{L_{H}^{2}}^{2}\right) \cdot \exp \left(\int_{\tau}^{t}\left(\frac{c^{2}}{2 c_{1} \mu_{1}}\|u(\xi)\|_{W}^{2}+\frac{L_{f}^{2}}{c_{1} \mu_{1}}\right) d \xi\right), \forall t \geq \tau .
$$

The proof of $(\mathbf{I})$ is complete.

(II) If $t \geq \tau+\nu$, then $t+\theta \geq \tau$ for any $\theta \in[-\nu, 0]$ and by (I), we have

$$
\begin{aligned}
\left\|u_{t}-v_{t}\right\|_{L_{H}^{2}}^{2} & =\int_{-\nu}^{0}\|u(t+\theta)-v(t+\theta)\|^{2} d \theta \\
& \leq \int_{-\nu}^{0} \sup _{\theta \in[-\nu, 0]}\|u(t+\theta)-v(t+\theta)\|^{2} d \theta \\
& \leq \nu\left(\frac{L_{f}^{2}}{c_{1} \mu_{1}}\|\psi-\varphi\|_{L_{H}^{2}}^{2}+\left\|u_{0}-v_{0}\right\|^{2}\right) \exp \left(\int_{\tau}^{t}\left(\frac{c^{2}}{2 c_{1} \mu_{1}}\|u(\xi)\|_{W}^{2}+\frac{L_{f}^{2}}{c_{1} \mu_{1}}\right) d \xi\right) .
\end{aligned}
$$

If $\tau \leq t<\tau+\nu$, we clearly have

$$
\begin{aligned}
\left\|u_{t}-v_{t}\right\|_{L_{H}^{2}}^{2}= & \int_{-\nu}^{0}\|u(t+\theta)-v(t+\theta)\|^{2} d \theta \\
\leq & \left(\left(\frac{\nu L_{f}^{2}}{c_{1} \mu_{1}}+1\right)\|\psi-\varphi\|_{L_{H}^{2}}^{2}+\nu\left\|u_{0}-v_{0}\right\|^{2}\right) \\
& \quad \quad \quad \exp \left(\int_{\tau}^{t}\left(\frac{c^{2}}{2 c_{1} \mu_{1}}\|u(\xi)\|_{W}^{2}+\frac{L_{f}^{2}}{c_{1} \mu_{1}}\right) d \xi\right) .
\end{aligned}
$$


Thus, for any $t \geq \tau$, there holds

$$
\begin{aligned}
\left\|u_{t}-v_{t}\right\|_{L_{H}^{2}}^{2} \leq & \left(\left(\frac{\nu L_{f}^{2}}{c_{1} \mu_{1}}+1\right)\|\psi-\varphi\|_{L_{H}^{2}}^{2}+\nu\left\|u_{0}-v_{0}\right\|^{2}\right) \\
& \times \exp \left(\int_{\tau}^{t}\left(\frac{c^{2}}{2 c_{1} \mu_{1}}\|u(\xi)\|_{W}^{2}+\frac{L_{f}^{2}}{c_{1} \mu_{1}}\right) d \xi\right) .
\end{aligned}
$$

Now, if $t \geq \tau+\nu$, then $t+\theta \geq \tau$ for any $\theta \in[-\nu, 0]$. By Theorem 2.1, we get

$$
\begin{aligned}
\left\|u_{t}-v_{t}\right\|_{\mathcal{C}_{H}}^{2} & =\max _{\theta \in[-\nu, 0]}\|u(t+\theta)-v(t+\theta)\|^{2} \\
& \leq\left(\frac{L_{f}^{2}}{c_{1} \mu_{1}}\|\psi-\varphi\|_{L_{H}^{2}}^{2}+\left\|u_{0}-v_{0}\right\|^{2}\right) \exp \left(\int_{\tau}^{t}\left(\frac{c^{2}}{2 c_{1} \mu_{1}}\|u(\xi)\|_{W}^{2}+\frac{L_{f}^{2}}{c_{1} \mu_{1}}\right) d \xi\right) .
\end{aligned}
$$

Hence (II) holds.

(III) If $\psi, \varphi \in \mathcal{C}_{H}$ and $u_{0}=\psi(0), v_{0}=\varphi(0)$, then by (I), for any $t \geq \tau$, we have

$$
\begin{aligned}
& \|u(t)-v(t)\|^{2} \\
\leq & \left(\frac{L_{f}^{2}}{c_{1} \mu_{1}}\|\psi-\varphi\|_{L_{H}^{2}}^{2}+\left\|u_{0}-v_{0}\right\|^{2}\right) \exp \left(\int_{\tau}^{t}\left(\frac{c^{2}}{2 c_{1} \mu_{1}}\|u(\xi)\|_{W}^{2}+\frac{L_{f}^{2}}{c_{1} \mu_{1}}\right) d \xi\right) \\
\leq & \left(\nu \frac{L_{f}^{2}}{c_{1} \mu_{1}}\|\psi-\varphi\|_{\mathcal{C}_{H}}^{2}+\|\psi-\varphi\|_{\mathcal{C}_{H}}^{2}\right) \exp \left(\int_{\tau}^{t}\left(\frac{c^{2}}{2 c_{1} \mu_{1}}\|u(\xi)\|_{W}^{2}+\frac{L_{f}^{2}}{c_{1} \mu_{1}}\right) d \xi\right) .
\end{aligned}
$$

Noticing that $u(t)-v(t)=\psi(t-\tau)-\varphi(t-\tau)$ when $\tau-\nu \leq t<\tau$, we get

$$
\|u(t)-v(t)\|^{2} \leq\left(\frac{\nu L_{f}^{2}}{c_{1} \mu_{1}}+1\right)\|\psi-\varphi\|_{\mathcal{C}_{H}}^{2} \exp \left(\int_{\tau}^{t}\left(\frac{c^{2}}{2 c_{1} \mu_{1}}\|u(\xi)\|_{W}^{2}+\frac{L_{f}^{2}}{c_{1} \mu_{1}}\right) d \xi\right)
$$

for $\forall t \geq \tau-\nu$. Therefore,

$$
\begin{aligned}
\left\|u_{t}-v_{t}\right\|_{\mathcal{C}_{H}}^{2} & =\max _{\theta \in[-\nu, 0]}\|u(t+\theta)-v(t+\theta)\|^{2} \\
& \leq\left(\frac{\nu L_{f}^{2}}{c_{1} \mu_{1}}+1\right)\|\psi-\varphi\|_{\mathcal{C}_{H}}^{2} \cdot \exp \left(\int_{\tau}^{t}\left(\frac{c^{2}}{2 c_{1} \mu_{1}}\|u(\xi)\|_{W}^{2}+\frac{L_{f}^{2}}{c_{1} \mu_{1}}\right) d \xi\right), \quad \forall t \geq \tau .
\end{aligned}
$$

The proof of Lemma 3.1 is now complete.

Note that from Lemma 3.1 one can directly deduce the uniqueness of solutions to problem (2.14). Thus, for each initial time $\tau \in \mathbb{R}$, Theorem 2.1 ensures that problem (2.14) admits a unique solution $u(\cdot)=u\left(\cdot ; \tau,\left(u_{0}, \psi\right)\right) \in L^{2}(\tau-\nu, T ; H) \cap L^{2}(\tau, T ; W) \cap$ $\mathcal{C}([\tau, T] ; H)$ for all $T>\tau$. We now can proceed as [19] did, with two different ways to construct the process associated to problem (2.14). Firstly, we can define a continuous process $\{\mathcal{U}(t, \tau)\}_{t \geq \tau}: \mathcal{C}_{H} \longmapsto \mathcal{C}_{H}$ as

$$
\mathcal{U}(t, \tau) \psi=u_{t}(\cdot ; \tau,(\psi(0), \psi)), \quad \forall \psi \in \mathcal{C}_{H} \text { and } t \geq \tau .
$$

Secondly, we can also define a continuous process $\{\mathcal{S}(t, \tau)\}_{t \geq \tau}: E_{H}^{2} \longmapsto E_{H}^{2}$ via

$$
\mathcal{S}(t, \tau)\left(u_{0}, \psi\right)=\left(u\left(t ; \tau,\left(u_{0}, \psi\right)\right), u_{t}\left(\cdot ; \tau,\left(u_{0}, \psi\right)\right)\right), \quad \forall\left(u_{0}, \psi\right) \in E_{H}^{2} \text { and } t \geq \tau .
$$

We next prove that $\{\mathcal{U}(t, \tau)\}_{t \geq \tau}$ and $\{\mathcal{S}(t, \tau)\}_{t \geq \tau}$ possess a family of uniformly bounded pullback absorbing sets in $\mathcal{C}_{H}$ and $E_{H}^{2}$, respectively. To this end, we introduce a family of mappings $\{\tilde{\mathcal{U}}(t, \tau)\}_{t \geq \tau}: E_{H}^{2} \longmapsto L_{H}^{2}$ defined by

$$
\tilde{\mathcal{U}}(t, \tau)\left(u_{0}, \psi\right)=u_{t}\left(\cdot ; \tau,\left(u_{0}, \psi\right)\right), \quad \forall\left(u_{0}, \psi\right) \in E_{H}^{2} \text { and } t \geq \tau .
$$


Then we have

$$
\mathcal{U}(t, \tau) \psi=\tilde{\mathcal{U}}(t, \tau)(\psi(0), \psi), \quad \forall \psi \in \mathcal{C}_{H} \text { and } t \geq \tau,
$$

and the process $\{\mathcal{S}(t, \tau)\}_{t \geq \tau}$ can be represented as

$$
\begin{aligned}
\mathcal{S}(t, \tau)\left(u_{0}, \psi\right) & =\left(u\left(t ; \tau,\left(u_{0}, \psi\right)\right), u_{t}\left(\cdot ; \tau,\left(u_{0}, \psi\right)\right)\right) \\
& \left.=\left(u\left(t ; \tau,\left(u_{0}, \psi\right)\right), \tilde{\mathcal{U}}(t, \tau)\left(u_{0}, \psi\right)\right)\right), \forall\left(u_{0}, \psi\right) \in E_{H}^{2}, t \geq \tau .
\end{aligned}
$$

Also, we define a linear mapping $J$ as

$$
J: \mathcal{C}_{H} \ni \psi \longmapsto J(\psi)=(\psi(0), \psi) \in H \times \mathcal{C}_{H} .
$$

Then $J$ is clearly continuous from $\mathcal{C}_{H}$ into $H \times \mathcal{C}_{H}$ and also into $E_{H}^{2}$. Since for all $\left(u_{0}, \psi\right) \in E_{H}^{2}$ it follows that $\mathcal{U}(t, \tau)\left(u_{0}, \psi\right)=u_{t}\left(\cdot ; \tau,\left(u_{0}, \psi\right)\right) \in \mathcal{C}_{H}$ provided $t \geq \tau+\nu$ (see Lemma $3.1(\mathbf{I I}))$, we then can rewrite $\mathcal{S}(t, \tau)\left(u_{0}, \psi\right)$ as

$$
\mathcal{S}(t, \tau)\left(u_{0}, \psi\right)=J\left(\tilde{\mathcal{U}}(t, \tau)\left(u_{0}, \psi\right)\right), \forall\left(u_{0}, \psi\right) \in E_{H}^{2}, t \geq \tau+\nu .
$$

As will be shown below, the above facts will help us to establish the estimates for the processes $\{\mathcal{U}(t, \tau)\}_{t \geq \tau}$ and $\{\mathcal{S}(t, \tau)\}_{t \geq \tau}$ in a direct way by using the obtained estimates for the mappings $\{\tilde{\mathcal{U}}(t, \tau)\}_{t \geq \tau}$.

The following definition and lemma were presented in 19 .

Definition 3.1. The family of bounded sets $\{\mathscr{B}(t)\}_{t \in \mathbb{R}} \subset \mathcal{C}_{H}$ is said to be pullback absorbing sets for $\{\tilde{\mathcal{U}}(t, \tau)\}_{t \geq \tau}$ in $E_{H}^{2}$ if for any given bounded set $\tilde{D} \subset E_{H}^{2}$ and each $t \in \mathbb{R}$, there exists $\tilde{T}_{\tilde{D}}(t)>0$ such that for any $s \geq \tilde{T}_{\tilde{D}}(t)$, it follows that $\tilde{\mathcal{U}}(t, t-s) \tilde{D} \subset \mathscr{B}(t)$.

Note that $\{\tilde{\mathcal{U}}(t, \tau)\}_{t \geq \tau}$ is not a process and the word "absorbing" in the above definition should be understood in a generalized sense.

LEMma $3.2([19])$. Let $\{\mathscr{B}(t)\}_{t \in \mathbb{R}} \subset \mathcal{C}_{H}$ be the family of bounded absorbing sets for $\{\tilde{\mathcal{U}}(t, \tau)\}_{t \geq \tau}$. Then

(i) $\{\mathscr{B}(t)\}_{t \in \mathbb{R}}$ is the family of pullback absorbing sets for $\{\mathcal{U}(t, \tau)\}_{t \geq \tau}$ in $\mathcal{C}_{H}$;

(ii) $\{J(\mathscr{B}(t))\}_{t \in \mathbb{R}}$ is the family of pullback absorbing sets for $\{\mathcal{S}(t, \tau)\}_{t \geq \tau}$ in $H \times \mathcal{C}_{H}$.

Lemma 3.3. Assume $\left(\mathbf{A}_{\mathbf{1}}\right)-\left(\mathbf{A}_{\mathbf{5}}\right)$ hold and $g \in H$. Then the family of mappings $\{\tilde{\mathcal{U}}(t, \tau)\}_{t \geq \tau}$ possesses a family of bounded pullback absorbing sets $\{\mathscr{B}(t)\}_{t \in \mathbb{R}} \subset \mathcal{C}_{H}$. Moreover, there exists a bounded set $\mathscr{B}_{1} \subset \mathcal{C}_{H}$ such that $\mathscr{B}(t)=\mathscr{B}_{1}$ for all $t \in \mathbb{R}$.

Proof. Let $\tilde{D} \subset E_{H}^{2}$ be bounded. Then there exists an $\tilde{r}>0$ such that

$$
\left\|u_{0}\right\|^{2}+\|\psi\|_{L_{H}^{2}}^{2} \leq \tilde{r}, \quad \forall\left(u_{0}, \psi\right) \in \tilde{D} .
$$

Take any $\left(u_{0}, \psi\right) \in \tilde{D}$ and $\tau \in \mathbb{R}$, and denote the corresponding solution as usual: $u(\cdot)=u\left(\cdot ; \tau,\left(u_{0}, \psi\right)\right)$. Multiplying the equation in (2.14) by $u(t)$ and using Lemma 2.1, we get

$$
\begin{aligned}
& \frac{1}{2} \frac{d}{d t}\|u(t)\|^{2}+2 c_{1} \mu_{1}\|u(t)\|_{W}^{2}+\langle B(u(t)), u(t)\rangle+\langle N(u(t)), u(t)\rangle \\
= & (g, u(t))+\left(f\left(t, u_{t}\right), u(t)\right) \\
\leq & \frac{1}{2 c_{1} \mu_{1}}\|g\|^{2}+\frac{3 c_{1} \mu_{1}}{2}\|u(t)\|^{2}+\frac{1}{4 c_{1} \mu_{1}}\left\|f\left(t, u_{t}\right)\right\|^{2} .
\end{aligned}
$$


Since $\langle B(u(t)), u(t)\rangle=0$, by the non-negativity of the term $\langle N(u(t)), u(t)\rangle$ and the fact that $\|\phi\|_{W} \geq\|\phi\|$ for all $\phi \in W$, we deduce from (3.40) that

$$
\frac{d}{d t}\|u(t)\|^{2}+c_{1} \mu_{1}\|u(t)\|^{2} \leq \frac{1}{c_{1} \mu_{1}}\|g\|^{2}+\frac{1}{2 c_{1} \mu_{1}}\left\|f\left(t, u_{t}\right)\right\|^{2} .
$$

Now by $\left(\mathbf{A}_{5}\right)$, we can choose a $\beta \in\left(0, \beta_{0}\right)$ such that

$$
\frac{L_{f}^{2}}{c_{1} \mu_{1}}+\beta<c_{1} \mu_{1}
$$

For the above $\beta$, we get by using (3.41) that

$$
\begin{aligned}
\frac{d}{d t}\left(e^{\beta t}\|u(t)\|^{2}\right) & =\beta e^{\beta t}\|u(t)\|^{2}+e^{\beta t} \frac{d}{d t}\|u(t)\|^{2} \\
& \leq \frac{1}{c_{1} \mu_{1}}\|g\|^{2} e^{\beta t}+\frac{\left\|f\left(t, u_{t}\right)\right\|^{2}}{2 c_{1} \mu_{1}} e^{\beta t}+\left(\beta-c_{1} \mu_{1}\right)\|u(t)\|^{2} e^{\beta t} .
\end{aligned}
$$

Integrating (3.43) over $[\tau, t]$, we can get

$$
\begin{aligned}
e^{\beta t}\|u(t)\|^{2}-e^{\beta \tau}\left\|u_{0}\right\|^{2} \leq & \frac{1}{c_{1} \mu_{1}} \int_{\tau}^{t}\|g\|^{2} e^{\beta \xi} d \xi+\int_{\tau}^{t} \frac{\left\|f\left(\xi, u_{\xi}\right)\right\|^{2}}{2 c_{1} \mu_{1}} e^{\beta \xi} d \xi \\
& +\left(\beta-c_{1} \mu_{1}\right) \int_{\tau}^{t}\|u(\xi)\|^{2} e^{\beta \xi} d \xi \\
= & \frac{\|g\|^{2}}{c_{1} \mu_{1} \beta} e^{\beta t}+\frac{L_{f}^{2}}{2 c_{1} \mu_{1}} \int_{\tau-\nu}^{\tau}\|\psi(\xi-\tau)\|^{2} e^{\beta \xi} d \xi \\
& \quad+\left(\frac{L_{f}^{2}}{2 c_{1} \mu_{1}}+\beta-c_{1} \mu_{1}\right) \int_{\tau}^{t}\|u(\xi)\|^{2} e^{\beta \xi} d \xi \\
\leq & \frac{\|g\|^{2}}{c_{1} \mu_{1} \beta} e^{\beta t}+\frac{L_{f}^{2} e^{\beta \tau}}{2 c_{1} \mu_{1}} \int_{-\nu}^{0}\|\psi(\theta)\|^{2} d \theta, \quad \forall t \geq \tau .
\end{aligned}
$$

Hence,

$$
e^{\beta t}\|u(t)\|^{2} \leq \frac{\|g\|^{2}}{c_{1} \mu_{1} \beta} e^{\beta t}+e^{\beta \tau} \tilde{r}\left(1+\frac{L_{f}^{2}}{2 c_{1} \mu_{1}}\right), \quad \forall t \geq \tau,
$$

and

$$
\|u(t)\|^{2} \leq \frac{\|g\|^{2}}{c_{1} \mu_{1} \beta}+\tilde{r}\left(1+\frac{L_{f}^{2}}{2 c_{1} \mu_{1}}\right) e^{-\beta(t-\tau)}, \quad \forall t \geq \tau .
$$

If $t \geq \tau+\nu$, then for any $\theta \in[-\nu, 0]$, we have $t+\theta \geq \tau$ and

$$
\|u(t+\theta)\|^{2} \leq \frac{\|g\|^{2}}{c_{1} \mu_{1} \beta}+\tilde{r}\left(1+\frac{L_{f}^{2}}{2 c_{1} \mu_{1}}\right) e^{\beta \nu} e^{-\beta(t-\tau)}, \quad \forall \theta \in[-\nu, 0] .
$$

Consequently,

$$
\left\|u_{t}\right\|_{\mathcal{C}_{H}}^{2}=\max _{\theta \in[-\nu, 0]}\|u(t+\theta)\|^{2} \leq \frac{\|g\|^{2}}{c_{1} \mu_{1} \beta}+\tilde{r}\left(1+\frac{L_{f}^{2}}{2 c_{1} \mu_{1}}\right) e^{\beta \nu} e^{-\beta(t-\tau)}, \forall t \geq \tau+\nu .
$$


We now consider the initial time $t-s$ instead of $\tau$, and then (3.45) implies

$$
\begin{aligned}
\left\|u_{t}\right\|_{\mathcal{C}_{H}}^{2} & =\left\|\tilde{\mathcal{U}}(t, t-s)\left(u_{0}, \psi\right)\right\|_{\mathcal{C}_{H}}^{2} \\
& \leq \frac{\|g\|^{2}}{c_{1} \mu_{1} \beta}+\tilde{r}\left(1+\frac{L_{f}^{2}}{2 c_{1} \mu_{1}}\right) e^{\beta \nu} e^{-\beta s}, \quad \forall t \in \mathbb{R}, s \geq \nu .
\end{aligned}
$$

Setting

$$
R_{H}^{2}=\frac{2\|g\|^{2}}{c_{1} \mu_{1} \beta}
$$

we deduce from (3.46) that there exists $\tilde{T}_{\tilde{D}}(t)=\tilde{T}_{\tilde{D}}$ (independent of $t$ ) such that for any $s \geq \tilde{T}_{\tilde{D}}$ and any $\left(u_{0}, \psi\right) \in \tilde{D} \subset E_{H}^{2}$,

$$
\left\|\tilde{\mathcal{U}}(t, t-s)\left(u_{0}, \psi\right)\right\|_{\mathcal{C}_{H}}^{2} \leq R_{H}^{2},
$$

that is to say,

$$
\{\mathscr{B}(t)\}_{t \in \mathbb{R}}=\mathscr{B}_{\mathcal{C}_{H}}\left(0, R_{H}\right) \doteq \mathscr{B}_{1},
$$

where $\mathscr{B}_{\mathcal{C}_{H}}\left(0, R_{H}\right)$ denotes the ball in $\mathcal{C}_{H}$ centered at zero with radius $R_{H}$, is the family of uniformly bounded pullback absorbing sets for the mappings $\{\tilde{\mathcal{U}}(t, \tau)\}_{t \geq \tau}$. The proof is complete.

As a corollary of Lemma 3.2 and Lemma 3.3, we have the following result.

Lemma 3.4. Assume $\left(\mathbf{A}_{\mathbf{1}}\right)-\left(\mathbf{A}_{\mathbf{5}}\right)$ hold and $g \in H$. Then the process $\{\mathcal{U}(t, \tau)\}_{t \geq \tau}$ possesses a family of uniformly bounded pullback absorbing sets $\left\{\mathscr{B}_{\mathcal{C}_{H}}(t)\right\}_{t \in \mathbb{R}} \subset \mathcal{C}_{H}$ and $\mathscr{B}_{\mathcal{C}_{H}}(t)=\mathscr{B}_{\mathcal{C}_{H}}\left(0, R_{H}\right)$ for all $t \in \mathbb{R}$. Also, the process $\{\mathcal{S}(t, \tau)\}_{t \geq \tau}$ possesses a family of uniformly bounded pullback absorbing sets $\left\{\mathscr{B}_{E_{H}^{2}}(t)\right\}_{t \in \mathbb{R}} \subset E_{H}^{2}$ and $\mathscr{B}_{E_{H}^{2}}(t)=$ $\mathscr{B}_{H}\left(0, R_{H}\right) \times \mathscr{B}_{L_{H}^{2}}\left(0, \nu^{1 / 2} R_{H}\right)$ for all $t \in \mathbb{R}$.

Similar to Lemma 3.3 , we next prove that the family of mappings $\{\tilde{\mathcal{U}}(t, \tau)\}_{t \geq \tau}$ possesses a family of uniformly bounded pullback absorbing sets in $\mathcal{C}_{W}$.

Lemma 3.5. Assume $\left(\mathbf{A}_{\mathbf{1}}\right)-\left(\mathbf{A}_{\mathbf{5}}\right)$ hold and $g \in H$. Then there exist positive constants $\tilde{R}_{W}, \tilde{\eta}_{1}$ and $\tilde{\eta}_{2}$ such that for any bounded set $\tilde{D} \subset E_{H}^{2}$,

$$
\begin{aligned}
& \left\|\tilde{\mathcal{U}}(t, t-s)\left(u_{0}, \psi\right)\right\|_{\mathcal{C}_{W}}^{2}=\max _{\theta \in[-\nu, 0]}\left\|u\left(t+\theta ; t-s,\left(u_{0}, \psi\right)\right)\right\|_{W}^{2} \leq \tilde{R}_{W}^{2}, \\
& \int_{t+\theta_{1}}^{t+\theta_{2}}\left\|A u\left(\xi ; t-s,\left(u_{0}, \psi\right)\right)\right\|^{2} d \xi \leq \tilde{\eta}_{1}\left|\theta_{1}-\theta_{2}\right|+\tilde{\eta}_{2},
\end{aligned}
$$

for all $s \geq \tilde{T}_{\tilde{D}}+\nu+1, t \in \mathbb{R},\left(u_{0}, \psi\right) \in E_{H}^{2}$, and $\theta_{1}, \theta_{2} \in[-\nu, 0]$.

Proof. Let $\tilde{D} \subset E_{H}^{2}$ be bounded. Then there exists $\tilde{r}>0$ such that

$$
\left\|u_{0}\right\|^{2}+\|\psi\|_{L_{H}^{2}}^{2} \leq \tilde{r}, \quad \forall\left(u_{0}, \psi\right) \in \tilde{D} .
$$

Take a fixed $t_{0} \in \mathbb{R}$ and $s \geq \tilde{T}_{\tilde{D}}$ (the absorbing time corresponding to the set $\mathscr{B}_{\mathcal{C}_{H}}\left(0, R_{H}\right)$ in Lemma 3.3), and consider the solution $u(\cdot)=u\left(\cdot ; t_{0}-s,\left(u_{0}, \psi\right)\right)$ for $\left(u_{0}, \psi\right) \in \tilde{D}$. 
Similar to (3.41), we have

$$
\frac{d}{d t}\|u(t)\|^{2}+c_{1} \mu_{1}\|u(t)\|_{W}^{2} \leq \frac{\|g\|^{2}}{c_{1} \mu_{1}}+\frac{1}{2 c_{1} \mu_{1}}\left\|f\left(t, u_{t}\right)\right\|^{2} .
$$

For $t \geq t_{0}$, we integrate (3.51) over $[t, t+1]$ to obtain

$$
\begin{aligned}
& \|u(t+1)\|^{2}-\|u(t)\|^{2}+c_{1} \mu_{1} \int_{t}^{t+1}\|u(\xi)\|_{W}^{2} d \xi \\
\leq & \frac{\|g\|^{2}}{c_{1} \mu_{1}}+\frac{L_{f}^{2}}{2 c_{1} \mu_{1}} \int_{t-\nu}^{t}\|u(\xi)\|^{2} d \xi+\frac{L_{f}^{2}}{2 c_{1} \mu_{1}} \int_{t}^{t+1}\|u(\xi)\|_{W}^{2} d \xi,
\end{aligned}
$$

and consequently, we have

$$
\begin{aligned}
\left(c_{1} \mu_{1}-\frac{L_{f}^{2}}{2 c_{1} \mu_{1}}\right) \int_{t}^{t+1}\|u(\xi)\|_{W}^{2} d \xi & \leq \frac{\|g\|^{2}}{c_{1} \mu_{1}}+\frac{L_{f}^{2}}{2 c_{1} \mu_{1}} \int_{t-\nu}^{t}\|u(\xi)\|^{2} d \xi+\|u(t)\|^{2} \\
& \leq \frac{\|g\|^{2}}{c_{1} \mu_{1}}+\frac{\nu \tilde{r} L_{f}^{2}}{2 c_{1} \mu_{1}}+R_{H}^{2}, \quad t \geq t_{0},
\end{aligned}
$$

and

$$
\int_{t}^{t+1}\|u(\xi)\|_{W}^{2} d \xi \leq \tilde{R}_{H}^{2}, \quad t \geq t_{0}
$$

where

$$
\tilde{R}_{H}^{2}=\frac{1}{c_{1} \mu_{1}-\frac{L_{f}^{2}}{2 c_{1} \mu_{1}}}\left(\frac{\|g\|^{2}}{c_{1} \mu_{1}}+\frac{\nu \tilde{r} L_{f}^{2}}{2 c_{1} \mu_{1}}+R_{H}^{2}\right) .
$$

We now multiply the equation in (2.14) by $A u$ and get for $\xi \geq t_{0}$ that

$$
\begin{gathered}
\frac{1}{2} \frac{\partial}{\partial \xi} a(u(\xi), u(\xi))+2 \mu_{1}\|A u(\xi)\|^{2}+\langle B(u(\xi)), A u(\xi)\rangle+\langle N(u(\xi)), A u(\xi)\rangle \\
=(g, A u(\xi))+\left(f\left(\xi, u_{\xi}\right), A u(\xi)\right) .
\end{gathered}
$$

We next evaluate the terms in (3.53). Firstly,

$$
\begin{aligned}
|(g, A u(\xi))|+\left|\left(f\left(\xi, u_{\xi}\right), A u(\xi)\right)\right| & \leq\|A u(\xi)\|\left(\|g\|+\left\|f\left(\xi, u_{\xi}\right)\right\|\right) \\
& \leq \frac{1}{\mu_{1}}\left(\|g\|^{2}+\left\|f\left(\xi, u_{\xi}\right)\right\|^{2}\right)+\frac{\mu_{1}}{2}\|A u(\xi)\|^{2} .
\end{aligned}
$$

Secondly, by the Gagliardo-Nirenberg inequality,

$$
\begin{aligned}
|\langle B(u(\xi)), A u(\xi)\rangle| & \leq c\|u(\xi)\|^{1 / 2}\|u(\xi)\|_{H^{1}(\Omega)}\|u(\xi)\|_{W}^{1 / 2}\|A u(\xi)\| \\
& \leq \frac{\mu_{1}}{2}\|A u(\xi)\|^{2}+\frac{c^{4}}{2 c_{1}^{2} \mu_{1}}\|u(\xi)\|^{2}\|u(\xi)\|_{H^{1}(\Omega)}^{4} .
\end{aligned}
$$

Thirdly, to estimate the term $\langle N(u(\xi)), A u(\xi)\rangle$, we set

$$
F(y)=2 \mu_{0}\left(\varepsilon+|y|^{2}\right)^{-\alpha / 2} y
$$

where

$$
y=\left(\begin{array}{ll}
y_{1} & y_{2} \\
y_{3} & y_{4}
\end{array}\right) \in \mathbb{R}^{4} \text { and }|y|^{2}=\sum_{i=1}^{4} y_{i}^{2}, \quad y_{i} \in \mathbb{R}, \quad i=1,2,3,4
$$


Then the first-order Fréchet derivative of $F(y)$ is

$$
D F(y)=2 \mu_{0}\left(\varepsilon+|y|^{2}\right)^{-\alpha / 2}\left(\begin{array}{cccc}
1-\frac{\alpha y_{1}^{2}}{\varepsilon+|y|^{2}} & -\frac{\alpha y_{1} y_{2}}{\varepsilon+|y|^{2}} & -\frac{\alpha y_{1} y_{3}}{\varepsilon+|y|^{2}} & -\frac{\alpha y_{1} y_{4}}{\varepsilon+|y|^{2}} \\
-\frac{\alpha y_{1} y_{2}}{\varepsilon+|y|^{2}} & 1-\frac{\alpha y_{2}^{2}}{\varepsilon+|y|^{2}} & -\frac{\alpha y_{2} y_{3}}{\varepsilon+|y|^{2}} & -\frac{\alpha y_{2} y_{4}}{\varepsilon+|y|^{2}} \\
-\frac{\alpha y_{1} y_{3}}{\varepsilon+|y|^{2}} & -\frac{\alpha y_{2} y_{3}}{\varepsilon+|y|^{2}} & 1-\frac{\alpha y_{3}^{2}}{\varepsilon+|y|^{2}} & -\frac{\alpha y_{3} y_{4}}{\varepsilon+|y|^{2}} \\
-\frac{\alpha y_{4} y_{4}}{\varepsilon+|y|^{2}} & -\frac{\alpha y_{2} y_{4}}{\varepsilon+|y|^{2}} & -\frac{\alpha y_{3} y_{4}}{\varepsilon+|y|^{2}} & 1-\frac{\alpha y_{4}^{2}}{\varepsilon+|y|^{2}}
\end{array}\right) .
$$

Since $0<\alpha<1$, we have

$$
\left|-\frac{\alpha y_{i} y_{j}}{\varepsilon+|y|^{2}}\right|<\left|\frac{y_{i} y_{j}}{\varepsilon+|y|^{2}}\right|<\frac{1}{\varepsilon}, \quad i, j=1,2,3,4,
$$

and

$$
0<1-\frac{\alpha y_{i}^{2}}{\varepsilon+|y|^{2}}<1, \quad i=1,2,3,4
$$

Consequently,

$$
\|D F(y)\| \leq 2 \mu_{0}\left(\varepsilon+|y|^{2}\right)^{-\alpha / 2} \sqrt{4+\frac{12}{\varepsilon^{2}}} \leq 2 \mu_{0} \varepsilon^{-\alpha / 2} \sqrt{4+\frac{12}{\varepsilon^{2}}}, \quad \forall y \in \mathbb{R}^{4} .
$$

Similarly, the second-order Fréchet derivative of $F(y)$ is a three-dimensional matrix

$$
D^{2} F(y)=\left(\frac{\partial^{2} F_{i}(y)}{\partial y_{j} \partial y_{k}}\right), \quad i=1,2,3,4, \quad j=1,2,3,4, \quad k=1,2,3,4,
$$

where $F_{i}(y)=2 \mu_{0}\left(\varepsilon+|y|^{2}\right)^{-\alpha / 2} y_{i}$. By some computations we see that the first-order and second-order Fréchet derivatives of $F(y)$ satisfy

$$
\|D F(y)\|+\left\|D^{2} F(y)\right\| \leq c_{3}\left(\mu_{0}, \varepsilon, \alpha\right) \doteq c_{3}, \quad \forall y \in \mathbb{R}^{4},
$$

where $c_{3}$ is a positive constant depending only on $\mu_{0}, \varepsilon$ and $\alpha$. For any $y, z \in \mathbb{R}^{4}$,

$$
F(y)-F(z)=\int_{0}^{1} D F(y+\tau(z-y))(z-y) d \tau .
$$

Taking $y=e(u)=\left(e_{i j}(u)\right), z=e(0)=\left(e_{i j}(0)\right)$, applying the integration by parts first and then the above inequality about $F(y)$, we have

$$
\begin{aligned}
|\langle N(u(\xi)), A u(\xi)\rangle| & =\left|-\int_{\Omega}\{\nabla \cdot[F(e(u(\xi)))-F(e(0))]\} \cdot A u(\xi) d x\right| \\
& \leq c_{3}\left(\|u(\xi)\|_{H^{1}(\Omega)}+\|u(\xi)\|_{W}\right)\|A u(\xi)\| \\
& \leq \frac{\mu_{1}}{2}\|A u(\xi)\|^{2}+\frac{c_{3}^{2}}{2 \mu_{1}}\|u(\xi)\|_{W}^{2} .
\end{aligned}
$$

It then follows from (3.53)-(3.57) and Lemma 2.1 that

$$
\begin{aligned}
& \frac{1}{2} \frac{\partial}{\partial \xi} a(u(\xi), u(\xi))+\frac{\mu_{1}}{2}\|A u(\xi)\|^{2} \\
\leq & \frac{1}{\mu_{1}}\left(\|g\|^{2}+\left\|f\left(\xi, u_{\xi}\right)\right\|^{2}\right)+\frac{c^{4}}{2 c_{1}^{2} \mu_{1}}\|u(\xi)\|^{2}\|u(\xi)\|_{H^{1}(\Omega)}^{4}+\frac{c_{3}^{2}}{2 \mu_{1}}\|u(\xi)\|_{W}^{2},
\end{aligned}
$$


whence, by Lemma 2.1 and the fact that $\|\phi\|_{H^{1}(\Omega)} \leq\|\phi\|_{W}$ for any $\phi \in W$, we get

$$
\begin{aligned}
& \frac{\partial}{\partial \xi} a(u(\xi), u(\xi)) \\
\leq & \frac{2\|g\|^{2}}{\mu_{1}}+\frac{2\left\|f\left(\xi, u_{\xi}\right)\right\|^{2}}{\mu_{1}}+\frac{c^{4}}{c_{1}^{2} \mu_{1}}\|u(\xi)\|^{2}\|u(\xi)\|_{W}^{4}+\frac{c_{3}^{2}}{\mu_{1}}\|u(\xi)\|_{W}^{2} \\
\leq & \frac{2\|g\|^{2}}{\mu_{1}}+\frac{2\left\|f\left(\xi, u_{\xi}\right)\right\|^{2}}{\mu_{1}}+\left(\frac{c^{4}\|u(\xi)\|^{2}}{c_{1}^{2} \mu_{1}}\|u(\xi)\|_{W}^{2}+\frac{c_{3}^{2}}{\mu_{1}}\right) \frac{a(u(\xi), u(\xi))}{c_{1}} .
\end{aligned}
$$

Noticing that $\xi \geq t_{0}$, we get by using $\left(\mathbf{A}_{3}\right)$ that

$$
\begin{aligned}
\frac{2\|g\|^{2}}{\mu_{1}}+\frac{2\left\|f\left(\xi, u_{\xi}\right)\right\|^{2}}{\mu_{1}} & \leq \frac{2\|g\|^{2}}{\mu_{1}}+\frac{2 C_{f}^{2}}{\mu_{1}}\|u(\xi)\|_{\mathcal{C}_{H}}^{2} \leq \frac{2\|g\|^{2}}{\mu_{1}}+\frac{2 C_{f}^{2}}{\mu_{1}} R_{H}^{2}, \\
\frac{c^{4}\|u(\xi)\|^{2}}{c_{1}^{2} \mu_{1}}\|u(\xi)\|_{W}^{2}+\frac{c_{3}^{2}}{\mu_{1}} & \leq \frac{c^{4} R_{H}^{2}}{c_{1}^{2} \mu_{1}}\|u(\xi)\|_{W}^{2}+\frac{c_{3}^{2}}{\mu_{1}} .
\end{aligned}
$$

Thus

$$
\int_{t}^{t+1}\left(\frac{2\|g\|^{2}}{\mu_{1}}+\frac{2\left\|f\left(\xi, u_{\xi}\right)\right\|^{2}}{\mu_{1}}\right) d \xi \leq \frac{2\|g\|^{2}}{\mu_{1}}+\frac{2 C_{f}^{2}}{\mu_{1}} R_{H}^{2} \doteq a_{1}, \quad \forall t \geq t_{0},
$$

and by (3.52), (3.61) and Lemma 2.1, we get

$$
\begin{aligned}
\frac{1}{c_{1}} \int_{t}^{t+1}\left(\frac{c^{4}\|u(\xi)\|^{2}}{c_{1}^{2} \mu_{1}}\|u(\xi)\|_{W}^{2}+\frac{c_{3}^{2}}{\mu_{1}}\right) d \xi & \leq \frac{c^{4} R_{H}^{2}}{c_{1}^{3} \mu_{1}} \int_{t}^{t+1}\|u(\xi)\|_{W}^{2} d \xi+\frac{c_{3}^{2}}{c_{1} \mu_{1}} \\
& \leq \frac{c^{4} R_{H}^{2} \tilde{R}_{H}^{2}}{c_{1}^{3} \mu_{1}}+\frac{c_{3}^{2}}{c_{1} \mu_{1}} \\
& \doteq a_{2}, \quad \forall t \geq t_{0}, \\
\int_{t}^{t+1} a(u(\xi), u(\xi)) d \xi & \leq \int_{t}^{t+1} c_{2}\|u(\xi)\|_{W}^{2} d \xi \\
& \leq c_{2} \tilde{R}_{H}^{2} \doteq a_{3}, \quad \forall t \geq t_{0} .
\end{aligned}
$$

Using the Uniform Gronwall Lemma (see Lemma 2.3) for $s \geq \tilde{T}_{\tilde{D}}$, we deduce from (3.59) and (3.62)-(3.64) that

$$
a(u(\xi), u(\xi)) \leq\left(a_{3}+a_{1}\right) e^{a_{2}}, \quad \forall \xi \geq t_{0}+1, \quad \forall s \geq \tilde{T}_{\tilde{D}},
$$

and again by Lemma 2.1,

$$
\|u(\xi)\|_{W}^{2} \leq \frac{1}{c_{1}} a(u(\xi), u(\xi)) \leq \frac{1}{c_{1}}\left(a_{3}+a_{1}\right) e^{a_{2}}, \quad \forall \xi \geq t_{0}+1, \quad \forall s \geq \tilde{T}_{\tilde{D}}
$$

Now if $s \geq \tilde{T}_{\tilde{D}}+\nu+1$, then (3.65) implies

$$
\sup _{\theta \in[-\nu, 0]}\left\|u\left(t_{0}+\theta\right)\right\|_{W}^{2} \leq \frac{1}{c_{1}}\left(a_{3}+a_{1}\right) e^{a_{2}} \doteq \tilde{R}_{W}^{2},
$$

where the constants $a_{1}, a_{2}, a_{3}$ and $c_{1}$ appearing in (3.66) are independent of the fixed time $t_{0} \in \mathbb{R}$. Thus (3.66) holds true for any $t_{0} \in \mathbb{R}$. We now denote $u_{t}(\cdot)=u_{t}\left(\cdot ; t-s,\left(u_{0}, \psi\right)\right)$. Theorem 2.1 ensures that $u_{t}(\cdot) \in \mathcal{C}_{W}$ for $\forall t \in \mathbb{R}$ provided $s>\nu$. Hence

$$
\left\|u_{t}\right\|_{\mathcal{C}_{W}}^{2} \leq \tilde{R}_{W}^{2}, \quad \forall t \in \mathbb{R}, \quad \forall s \geq \tilde{T}_{\tilde{D}}+\nu+1,
$$


and (3.49) holds. We next prove (3.50). Indeed, we deduce from (3.58) that

$$
\begin{gathered}
\|A u(\xi)\|^{2} \leq \frac{2\|g\|^{2}}{\mu_{1}^{2}}+\frac{2\left\|f\left(\xi, u_{\xi}\right)\right\|^{2}}{\mu_{1}^{2}}+\frac{c^{4}}{c_{1}^{2} \mu_{1}^{2}}\|u(\xi)\|^{2}\|u(\xi)\|_{H^{1}(\Omega)}^{4} \\
+\frac{c_{3}^{2}}{2 \mu_{1}^{2}}\|u(\xi)\|_{V}^{2}-\frac{1}{\mu_{1}} \frac{\partial}{\partial \xi} a(u(\xi), u(\xi)) .
\end{gathered}
$$

Choose $s \geq \tilde{T}_{\tilde{D}}+\nu+1$ and $\theta_{1}, \theta_{2} \in[-\nu, 0]$ (let $\theta_{2}>\theta_{1}$ without loss of generality). Then we get

$$
\begin{aligned}
& \int_{t+\theta_{1}}^{t+\theta_{2}}\|A u(\xi)\|^{2} d \xi \\
\leq & \frac{2\|g\|^{2}}{\mu_{1}^{2}}\left|\theta_{2}-\theta_{1}\right|+\frac{2}{\mu_{1}^{2}} \int_{t+\theta_{1}}^{t+\theta_{2}}\left\|f\left(\xi, u_{\xi}\right)\right\|^{2} d \xi+\frac{c^{4}}{c_{1}^{2} \mu_{1}^{2}} \int_{t+\theta_{1}}^{t+\theta_{2}}\|u(\xi)\|^{2}\|u(\xi)\|_{H^{1}(\Omega)}^{4} d \xi \\
& +\frac{c_{3}^{2}}{2 \mu_{1}^{2}} \int_{t+\theta_{1}}^{t+\theta_{2}}\|u(\xi)\|_{W}^{2} d \xi+\frac{1}{\mu_{1}} a\left(u\left(t+\theta_{1}\right), u\left(t+\theta_{1}\right)\right) \\
\leq & \frac{2\|g\|^{2}}{\mu_{1}^{2}}\left|\theta_{2}-\theta_{1}\right|+\frac{2 C_{f}^{2}}{\mu_{1}^{2}} \int_{t+\theta_{1}}^{t+\theta_{2}}\left\|u_{\xi}\right\|_{\mathcal{C}_{H}}^{2} d \xi+\frac{c^{4} R_{H}^{2}}{c_{1}^{2} \mu_{1}^{2}} \int_{t+\theta_{1}}^{t+\theta_{2}}\|u(\xi)\|_{H^{1}(\Omega)}^{4} d \xi \\
+\frac{c_{3}^{2} \tilde{R}_{W}^{2}}{2 \mu_{1}^{2}}\left|\theta_{2}-\theta_{1}\right|+\frac{c_{2}}{\mu_{1}}\left\|u\left(t+\theta_{1}\right)\right\|_{W}^{2} & \left(\frac{2\|g\|^{2}}{\mu_{1}^{2}}+\frac{2 C_{f}^{2} R_{H}^{2}}{\mu_{1}^{2}}+\frac{c^{4} R_{H}^{2} \tilde{R}_{W}^{4}}{c_{1}^{2} \mu_{1}^{2}}+\frac{c_{3}^{2} \tilde{R}_{W}^{2}}{2 \mu_{1}^{2}}\right)\left|\theta_{2}-\theta_{1}\right|+\frac{c_{2}}{\mu_{1}} \tilde{R}_{W}^{2} .
\end{aligned}
$$

The proof of Lemma 3.5 is now complete.

Lemma 3.6. Assume $\left(\mathbf{A}_{\mathbf{1}}\right)-\left(\mathbf{A}_{\mathbf{5}}\right)$ hold and $g \in H$. Then there exist positive constants $R_{W}, \eta_{1}$ and $\eta_{2}$ such that for any bounded set $\mathscr{B} \subset \mathcal{C}_{H}$ and for $T_{\mathscr{B}}=\tilde{T}_{J(\mathscr{B})}$ (the absorbing time corresponding to the set $\mathscr{B}_{\mathcal{C}_{H}}\left(0, R_{H}\right)$ in Lemma 3.3), the following holds:

$$
\begin{aligned}
\|\mathcal{U}(t, t-s) \psi\|_{\mathcal{C}_{W}}^{2} & =\left\|u_{t}(\cdot ; t-s, J(\psi))\right\|_{\mathcal{C}_{W}}^{2} \\
& =\max _{\theta \in[-\nu, 0]}\|u(t+\theta ; t-s, J(\psi))\|_{W}^{2} \leq R_{W}^{2}, \\
\int_{t+\theta_{1}}^{t+\theta_{2}}\|A u(\xi ; t-s, J(\psi))\|^{2} d \xi & \leq \eta_{1}\left|\theta_{1}-\theta_{2}\right|+\eta_{2},
\end{aligned}
$$

for all $s \geq T_{\mathscr{B}}+\nu+1, t \in \mathbb{R}, \psi \in \mathscr{B}$, and $\theta_{1}, \theta_{2} \in[-\nu, 0]$. Moreover, the two families of sets $\left\{\mathscr{B}_{\mathcal{C}_{W}}(t)\right\}_{t \in \mathbb{R}} \subset \mathcal{C}_{W},\left\{\mathscr{B}_{E_{W}^{2}}(t)\right\}_{t \in \mathbb{R}} \subset E_{W}^{2}$, where $\mathscr{B}_{\mathcal{C}_{W}}(t)=\mathscr{B}_{\mathcal{C}_{W}}\left(0, R_{W}\right), \mathscr{B}_{E_{W}^{2}}(t)=$ $\mathscr{B}_{W}\left(0, R_{W}\right) \times \mathscr{B}_{L_{W}^{2}}\left(0, \nu^{1 / 2} R_{W}\right)$ for all $t \in \mathbb{R}$, are uniformly bounded pullback absorbing sets of the processes $\{\mathcal{U}(t, \tau)\}_{t \geq \tau}$ in $\mathcal{C}_{W}$ and $\{\mathcal{S}(t, \tau)\}_{t \geq \tau}$ in $E_{W}^{2}$, respectively.

Proof. First, (3.68) and (3.69) can be deduced directly from (3.49) and (3.50). Then (3.68) implies that the family of sets $\left\{\mathscr{B}_{\mathcal{C}_{W}}(t)\right\}_{t \in \mathbb{R}} \subset \mathcal{C}_{W}$, where $\mathscr{B}_{\mathcal{C}_{W}}(t)=\mathscr{B}_{\mathcal{C}_{W}}\left(0, R_{W}\right)$ (the ball in $\mathcal{C}_{W}$ centered at zero with radius $R_{W}$ ), $t \in \mathbb{R}$, are pullback absorbing for $\{\mathcal{U}(t, \tau)\}_{t \geq \tau}$ in $\mathcal{C}_{W}$. Finally, note that $\left\{J\left(\mathscr{B}_{\mathcal{C}_{W}}(t)\right)\right\}_{t \in \mathbb{R}}$ is a family of uniformly bounded pullback absorbing sets for $\{\mathcal{S}(t, \tau)\}_{t \geq \tau}$ in $E_{W}^{2}$. At the same time, $\|\psi\|_{L_{W}^{2}}^{2} \leq \nu\|\psi\|_{\mathcal{C}_{W}}$ for any $\psi \in \mathcal{C}_{W}$, and $J\left(\mathscr{B}_{\mathcal{C}_{W}}(t)\right)=\left\{(\psi(0), \psi): \psi \in \mathscr{B}_{\mathcal{C}_{W}}\left(0, R_{W}\right)\right\}$. It then follows 
that $J\left(\mathscr{B}_{\mathcal{C}_{W}}(t)\right) \subset \mathscr{B}_{W}\left(0, R_{W}\right) \times \mathscr{B}_{L_{W}^{2}}\left(0, \nu^{1 / 2} R_{W}\right) \doteq \mathscr{B}_{E_{W}^{2}}(t), t \in \mathbb{R}$, where $\mathscr{B}_{W}\left(0, R_{W}\right)$ denotes the ball in $W$ centered at zero with radius $R_{W}$ and $\mathscr{B}_{L_{W}^{2}}\left(0, \nu^{1 / 2} R_{W}\right)$ denotes the ball in $L_{W}^{2}$ centered at zero with radius $\nu^{1 / 2} R_{W}$.

At this stage, we can state and prove the main result of this section, which reads as

Theorem 3.1. Let $\left(\mathbf{A}_{\mathbf{1}}\right)-\left(\mathbf{A}_{\mathbf{5}}\right)$ hold and $g \in H$. Then the process $\{\mathcal{U}(t, \tau)\}_{t \geq \tau}$ possesses a unique uniformly bounded pullback attractor $\left\{\mathscr{A}_{\mathcal{C}_{H}}(t)\right\}_{t \in \mathbb{R}} \subset \mathscr{B}_{\mathcal{C}_{W}}\left(0, R_{W}\right) \subset \mathcal{C}_{H}$ and the process $\{\mathcal{S}(t, \tau)\}_{t \geq \tau}$ possesses a unique uniformly bounded pullback attractor $\left\{\mathscr{A}_{E_{H}^{2}}(t)\right\}_{t \in \mathbb{R}} \subset \mathscr{B}_{W}\left(0, R_{W}\right) \times \mathscr{B}_{L_{W}^{2}}\left(0, \nu^{1 / 2} R_{W}\right) \subset E_{H}^{2}$. Furthermore, $\mathscr{A}_{E_{H}^{2}}(t) \subset H \times \mathcal{C}_{H}$ for all $t \in \mathbb{R}$, and the two attractors are related via

$$
\mathscr{A}_{E_{H}^{2}}(t)=J\left(\mathscr{A}_{\mathcal{C}_{H}}(t)\right), \quad \forall t \in \mathbb{R} .
$$

Proof. Let $\left\{\mathscr{B}_{\mathcal{C}_{W}}(t)\right\}_{t \in \mathbb{R}}$ be defined as in Lemma 3.6. Then $\left\{\mathscr{B}_{\mathcal{C}_{W}}(t)\right\}_{t \in \mathbb{R}}$ is a family of uniformly bounded pullback absorbing sets for $\{\mathcal{U}(t, \tau)\}_{t \geq \tau}$ in $\mathcal{C}_{W}$. For simplicity, write $\mathscr{B}_{2}=\mathscr{B}_{\mathcal{C}_{W}}\left(0, R_{W}\right)$ and set $\tilde{\mathscr{B}}_{2}=J\left(\mathscr{B}_{2}\right)$. Then Lemma 3.5 shows that there exists $T_{\mathscr{B}_{2}}=\tilde{T}_{\tilde{\mathscr{B}}_{2}}+\nu+1$ such that

$$
\tilde{\mathcal{U}}(t, t-s) \tilde{\mathscr{B}}_{2} \subset \mathscr{B}_{2}, \quad \forall t \in \mathbb{R} \text { and } s \geq T_{\mathscr{B}_{2}} .
$$

Writing

$$
\mathscr{B}_{3}(t)=\bigcup_{s \geq T_{\mathscr{B}_{2}}} \tilde{\mathcal{U}}(t, t-s) \tilde{\mathscr{B}}_{2} \subset \mathscr{B}_{2} \subset \mathcal{C}_{W}, \quad t \in \mathbb{R},
$$

we see that $\left\{\mathscr{B}_{3}(t)\right\}_{t \in \mathbb{R}}$ is a family of uniformly bounded pullback absorbing sets of $\{\tilde{\mathcal{U}}(t, \tau)\}_{t \geq \tau}$. We next use the Ascoli-Arzelà theorem to prove that for each $t \in \mathbb{R}, \mathscr{B}_{3}(t)$ is relatively compact in $\mathcal{C}_{H}$. To this end, we need to check the following two points.

(1) For each $t \in \mathbb{R}, \mathscr{B}_{3}(t)$ is equi-continuous in $\mathcal{C}_{H}$; that is, given any $\epsilon>0, \exists \delta>0$ such that if $\left|\theta_{2}-\theta_{1}\right| \leq \delta$, then $\left\|\tilde{\mathcal{U}}(t, t-s) J(\psi)\left(\theta_{2}\right)-\tilde{\mathcal{U}}(t, t-s) J(\psi)\left(\theta_{1}\right)\right\| \leq \epsilon$, for $\forall s \geq \tilde{T}_{\mathscr{B}_{2}}$ and $\forall \psi \in \mathscr{B}_{2}$.

(2) For each $t \in \mathbb{R}$ and $\forall \theta \in[-\nu, 0]$,

$$
\bigcup_{s \geq \tilde{T}} \bigcup_{\psi \in \mathscr{B}_{2}} \tilde{\mathcal{U}}(t, t-s)(J(\psi))(\theta) \text { is pre-compact in } H \text {. }
$$

Indeed, on the one hand, we have

$$
\begin{aligned}
& \left\|\tilde{\mathcal{U}}(t, t-s)(J(\psi))\left(\theta_{2}\right)-\tilde{\mathcal{U}}(t, t-s)(J(\psi))\left(\theta_{1}\right)\right\| \\
& =\| u\left(t+\theta_{2}, t-s,(J(\psi))-u\left(t+\theta_{1}, t-s,(J(\psi)) \|\right.\right.
\end{aligned}
$$

for $\forall t \in \mathbb{R}, s \geq T_{\mathscr{B}_{2}}$ and $\forall \psi \in \mathscr{B}_{2}, \theta_{1}, \theta_{2} \in[-\nu, 0]$. Denoting $u(\cdot)=u(\cdot ; t-s, J(\psi))$ and assuming (without loss of generality) $\theta_{2}>\theta_{1}$, we obtain

$$
\begin{aligned}
& \left\|u\left(t+\theta_{2}\right)-u\left(t+\theta_{1}\right)\right\| \\
= & \left\|\int_{t+\theta_{1}}^{t+\theta_{2}} \frac{\partial}{\partial \xi} u(\xi) d \xi\right\| \leq \int_{t+\theta_{1}}^{t+\theta_{2}}\left\|\frac{\partial}{\partial \xi} u(\xi)\right\| d \xi \\
\leq & \int_{t+\theta_{1}}^{t+\theta_{2}}\left(\left\|2 \mu_{1} A u(\xi)\right\|+\|B(u(\xi))\|+\|N(u(\xi))\|+\|g\|+\left\|f\left(\xi, u_{\xi}\right)\right\|\right) d \xi .
\end{aligned}
$$


By the Gagliardo-Nirenberg inequality, we get

$$
\begin{aligned}
|\langle B(u(\xi)), \phi\rangle| & \leq \sum_{i, j=1}^{2}\left|\int_{\Omega} u_{i} \frac{\partial u_{i}}{\partial x_{j}} \phi_{j} d x\right| \leq\|u\|_{L^{4}(\Omega)}\|\nabla u\|_{L^{4}(\Omega)}\|\phi\| \\
& \leq c\|u\|\|\Delta u\|\|\phi\|, \quad \forall \phi \in H,
\end{aligned}
$$

and thus by using (2.8), we obtain

$$
\begin{aligned}
\int_{t+\theta_{1}}^{t+\theta_{2}}\|B(u(\xi))\| d \xi & \leq c R_{H} \int_{t+\theta_{1}}^{t+\theta_{2}}\|\Delta u(\xi)\| d \xi \leq c R_{H} \int_{t+\theta_{1}}^{t+\theta_{2}}\|u(\xi)\|_{W} d \xi \\
& \leq \frac{c R_{H}}{c_{1}} \int_{t+\theta_{1}}^{t+\theta_{2}}\|A u(\xi)\| d \xi, \quad s \geq T_{\mathscr{B}_{2}} .
\end{aligned}
$$

Analogously, applying the same derivations to (3.57), we have

$$
\int_{t+\theta_{1}}^{t+\theta_{2}}\|N(u(\xi))\| d \xi \leq c_{3} \int_{t+\theta_{1}}^{t+\theta_{2}}\|\Delta u(\xi)\| d \xi \leq \frac{c_{3}}{c_{1}} \int_{t+\theta_{1}}^{t+\theta_{2}}\|A u(\xi)\| d \xi, \quad s \geq T_{\mathscr{B}_{2}} .
$$

It then follows from (3.69) and (3.71) $-(3.73)$ that

$$
\begin{aligned}
& \left\|u\left(t+\theta_{2}\right)-u\left(t+\theta_{1}\right)\right\| \\
\leq & \|g\|\left|\theta_{1}-\theta_{2}\right|+\int_{t+\theta_{1}}^{t+\theta_{2}}\left\|f\left(\xi, u_{\xi}\right)\right\| d \xi+\left(2 \mu_{1}+\frac{c R_{H}}{c_{1}}+\frac{c_{3}}{c_{1}}\right) \int_{t+\theta_{1}}^{t+\theta_{2}}\|A u(\xi)\| d \xi \\
\leq & \left(\|g\|+C_{f} R_{H}\right)\left|\theta_{1}-\theta_{2}\right| \\
& \quad+\left(2 \mu_{1}+\frac{c R_{H}}{c_{1}}+\frac{c_{3}}{c_{1}}\right)\left|\theta_{1}-\theta_{2}\right|^{1 / 2}\left(\int_{t+\theta_{1}}^{t+\theta_{2}}\|A u(\xi)\|^{2} d \xi\right)^{1 / 2} \\
\leq & \left(\|g\|+C_{f}^{2} R_{H}\right)\left|\theta_{1}-\theta_{2}\right|+\left(2 \mu_{1}+\frac{c R_{H}}{c_{1}}+\frac{c_{3}}{c_{1}}\right)\left|\theta_{1}-\theta_{2}\right|^{1 / 2}\left(\eta_{1}\left|\theta_{1}-\theta_{2}\right|+\eta_{2}\right)^{1 / 2},
\end{aligned}
$$

which gives the desired equi-continuity, i.e. (1) holds. On the other hand, for any fixed $\theta \in[-\nu, 0]$ and $t \in \mathbb{R}$, the set

$$
\left\{u(t+\theta ; t-s, J(\psi)): s \geq T_{\mathscr{B}_{2}}, \quad \psi \in \mathscr{B}_{2}\right\}
$$

is pre-compact in $H$ because it is bounded in $W$ (see (3.49) $)$ and the embedding $W \hookrightarrow H$ is compact. Thus (2) holds. Therefore, $\left\{\mathscr{B}_{3}(t)\right\}_{t \in \mathbb{R}}$ is a family of compact (uniformly bounded) pullback absorbing sets for $\{\tilde{\mathcal{U}}(t, \tau)\}_{t \geq \tau}$ in $\mathcal{C}_{H}$. Then Lemma 3.2 ensures that $\left\{\mathscr{B}_{3}(t)\right\}_{t \in \mathbb{R}}$ and $\left\{J\left(\mathscr{B}_{3}(t)\right)\right\}_{t \in \mathbb{R}}$ are respectively the families of compact (uniformly bounded) pullback absorbing sets for $\{\mathcal{U}(t, \tau)\}_{t \geq \tau}$ in $\mathcal{C}_{H}$ and for $\{\mathcal{S}(t, \tau)\}_{t \geq \tau}$ in $E_{H}^{2}$, respectively. Thus the existence of the uniformly bounded pullback attractors $\left\{\mathscr{A}_{\mathcal{C}_{H}}(t)\right\}_{t \in \mathbb{R}}$ for $\{\mathcal{U}(t, \tau)\}_{t \geq \tau}$ in $\mathcal{C}_{H}$ and $\left\{\mathscr{A}_{E_{H}^{2}}(t)\right\}_{t \in \mathbb{R}}$ for $\{\mathcal{S}(t, \tau)\}_{t \geq \tau}$ in $E_{H}^{2}$ follows, respectively.

We next prove (3.70). By (3.36), (3.39) and the invariance property of the pullback attractor, we have for $\forall t \geq \tau$ that

$$
\begin{aligned}
\mathcal{S}(t, \tau) J\left(\mathscr{A}_{\mathcal{C}_{H}}(\tau)\right) & =J\left(\tilde{\mathcal{U}}(t, \tau) J\left(\mathscr{A}_{\mathcal{C}_{H}}(\tau)\right)\right)=J\left(\mathcal{U}(t, \tau) \mathscr{A}_{\mathcal{C}_{H}}(\tau)\right) \\
& =J\left(\mathscr{A}_{\mathcal{C}_{H}}(t)\right) .
\end{aligned}
$$


Since $\left\{\mathscr{A}_{\mathcal{C}_{H}}(t)\right\}_{t \in \mathbb{R}} \subset \mathscr{B}_{2}$ are uniformly (with respect to $t \in \mathbb{R}$ ) bounded in $\mathcal{C}_{H}$, $\left\{J\left(\mathscr{A}_{\mathcal{C}_{H}}(t)\right)\right\}_{t \in \mathbb{R}}$ are uniformly (with respect to $t \in \mathbb{R}$ ) bounded in $E_{H}^{2}$. Then by the pullback attracting property of the pullback attractor and (3.74), we get

$$
\begin{aligned}
& \operatorname{Dist}_{E_{H}^{2}}\left(J\left(\mathscr{A}_{\mathcal{C}_{H}}(t)\right), \mathscr{A}_{E_{H}^{2}}(t)\right) \\
= & \operatorname{Dist}_{E_{H}^{2}}\left(\mathcal{S}(t, t-s) J\left(\mathscr{A}_{\mathcal{C}_{H}}(t-s)\right), \mathscr{A}_{E_{H}^{2}}(t)\right) \quad(\forall s>0) \\
= & \lim _{s \rightarrow+\infty} \operatorname{Dist}_{E_{H}^{2}}\left(\mathcal{S}(t, t-s) J\left(\mathscr{A}_{\mathcal{C}_{H}}(t-s)\right), \mathscr{A}_{E_{H}^{2}}(t)\right) \\
= & 0,
\end{aligned}
$$

and thus

$$
J\left(\mathscr{A}_{\mathcal{C}_{H}}(t)\right) \subset \mathscr{A}_{E_{H}^{2}}(t), \quad \forall t \in \mathbb{R} .
$$

Noticing that $\left\{J\left(\mathscr{A}_{\mathcal{C}_{H}}(t)\right\}_{t \in \mathbb{R}}\right.$ is the family of uniformly pullback attracting sets for $\{\mathcal{S}(t, \tau)\}_{t \geq \tau}$ in $E_{H}^{2}$ and $\left\{\mathscr{A}_{E_{H}^{2}}(t)\right\}_{t \in \mathbb{R}}$ are uniformly (with respect to $t \in \mathbb{R}$ ) bounded in $E_{H}^{2}$, we have, also using the invariance property and pullback attracting property of the pullback attractor,

$$
\begin{aligned}
& \operatorname{Dist}_{E_{H}^{2}}\left(\mathscr{A}_{E_{H}^{2}}(t), J\left(\mathscr{A}_{\mathcal{C}_{H}}(t)\right)\right) \\
= & \operatorname{Dist}_{E_{H}^{2}}\left(\mathcal{S}(t, t-s) \mathscr{A}_{E_{H}^{2}}(t-s), J\left(\mathscr{A}_{\mathcal{C}_{H}}(t)\right)\right) \quad(\forall s>0) \\
= & \lim _{s \rightarrow+\infty} \operatorname{Dist}_{E_{H}^{2}}\left(\mathcal{S}(t, t-s) \mathscr{A}_{E_{H}^{2}}(t-s), J\left(\mathscr{A}_{\mathcal{C}_{H}}(t)\right)\right) \\
= & 0,
\end{aligned}
$$

and hence $\mathscr{A}_{E_{H}^{2}}(t) \subset J\left(\mathscr{A}_{\mathcal{C}_{H}}(t)\right), \forall t \in \mathbb{R}$, by which and (3.75) we get (3.70). The proof of Theorem 3.1 is complete.

4. Existence of a pullback attractor in $E_{W}^{2}$. In this section, we establish that the process $\{\mathcal{S}(t, \tau)\}_{t \geq \tau}$ possesses a uniform bounded pullback attractor $\left\{\mathscr{A}_{E_{W}^{2}}(t)\right\}_{t \in \mathbb{R}} \subset$ $E_{W}^{2}$. The main step is to prove that the family of uniformly bounded absorbing sets $\left\{\mathscr{B}_{E_{W}^{2}}(t)\right\}_{t \in \mathbb{R}}$ obtained in Lemma 3.6 is compact in $E_{W}^{2}$. The argument used here is the energy equality method, which is due to J. M. Ball (see, e.g., [5]). The energy equality method has been proved to be very useful for the investigation of the asymptotic compactness of dynamical systems, especially for the case that the spatial domain is unbounded (see, e.g., [31, 49, 56]).

By Theorem 2.1 (2), we can also define a continuous process $\{\mathcal{S}(t, \tau)\}_{t \geq \tau}: E_{W}^{2} \longmapsto E_{W}^{2}$ via

$$
\mathcal{S}(t, \tau)\left(u_{0}, \psi\right)=\left(u\left(t ; \tau,\left(u_{0}, \psi\right)\right), u_{t}\left(\cdot ; \tau,\left(u_{0}, \psi\right)\right)\right), \quad \forall\left(u_{0}, \psi\right) \in E_{W}^{2} \text { and } t \geq \tau .
$$

Definition 4.1. The process $\{\mathcal{S}(t, \tau)\}_{t \geq \tau}$ is said to be pullback asymptotically compact in $E_{W}^{2}$ if for each $t \in \mathbb{R}$, any bounded sequence $\left\{\left(u_{0}^{(n)}, \psi^{(n)}\right)\right\}$ of $E_{W}^{2}$, and $\left\{s_{n}\right\} \subset \mathbb{R}_{+}$ with $s_{n} \rightarrow+\infty$ as $n \rightarrow \infty$, the sequence $\left\{\mathcal{S}\left(t, t-s_{n}\right)\left(u_{0}^{(n)}, \psi^{(n)}\right)\right\}$ has a convergent subsequence. 
Lemma 4.1. Assume $\left(\mathbf{A}_{\mathbf{1}}\right)-\left(\mathbf{A}_{\mathbf{5}}\right)$ hold and $g \in H$. Then the process $\{\mathcal{S}(t, \tau)\}_{t \geq \tau}$ is pullback asymptotically compact in $E_{W}^{2}$.

Proof. By Lemma 3.6, we only need to prove

$$
\left\{\begin{array}{l}
\text { For each } t \in \mathbb{R}, \text { any }\left\{s_{n}\right\} \subset \mathbb{R}_{+} \text {with } s_{n} \rightarrow+\infty \text { as } n \rightarrow \infty \text { and } \\
\text { any }\left\{\left(u_{0}^{(n)}, \psi^{(n)}\right)\right\} \subset \mathscr{B}_{W}\left(0, R_{W}\right) \times \mathscr{B}_{L_{W}^{2}}\left(0, \nu^{1 / 2} R_{W}\right), \text { the sequence } \\
\left\{\mathcal{S}\left(t, t-s_{n}\right)\left(u_{0}^{(n)}, \psi^{(n)}\right)\right\} \text { has a convergent subsequence. }
\end{array}\right.
$$

For brevity, we let $\left\{s_{n}\right\} \subset\left[T^{*},+\infty\right)$, where

$$
T^{*}=T_{\mathscr{B}_{W}}\left(0, R_{W}\right) \times \mathscr{B}_{L_{W}^{2}}\left(0, \nu^{1 / 2} R_{W}\right)
$$

denotes the corresponding absorbing time for $\mathscr{B}_{W}\left(0, R_{W}\right) \times \mathscr{B}_{L_{W}^{2}}\left(0, \nu^{1 / 2} R_{W}\right)$ in Lemma 3.6. Then $\left\{\mathcal{S}\left(t, t-s_{n}\right)\left(u_{0}^{(n)}, \psi^{(n)}\right)\right\} \subset \mathscr{B}_{W}\left(0, R_{W}\right) \times \mathscr{B}_{L_{W}^{2}}\left(0, \nu^{1 / 2} R_{W}\right) \subset W \times L_{W}^{2}$. Note that $W \times L_{W}^{2}$ is a reflexive Banach space. So there exists a subsequence (still denoted by $\left.\left\{\mathcal{S}\left(t, t-s_{n}\right)\left(u_{0}^{(n)}, \psi^{(n)}\right)\right\}\right)$ such that

$$
\mathcal{S}\left(t, t-s_{n}\right)\left(u_{0}^{(n)}, \psi^{(n)}\right) \rightarrow\left(w_{0}, \psi\right) \text { weakly in } W \times L_{W}^{2} \text { as } n \rightarrow \infty
$$

for some $\left(w_{0}, \psi\right) \in W \times L_{W}^{2}$. Similarly, for each $T \in \mathbb{N}\left(T<s_{n}\right.$ for $n$ being large enough), we have

$$
\mathcal{S}\left(t-T, t-s_{n}\right)\left(u_{0}^{(n)}, \psi^{(n)}\right) \rightarrow\left(w_{T}, \psi_{T}\right) \text { weakly in } W \times L_{W}^{2} \text { as } n \rightarrow \infty
$$

for some $\left(w_{T}, \psi_{T}\right) \in W \times L_{W}^{2}$. By the property of the process, we have

$$
\begin{aligned}
& \mathcal{S}\left(t, t-s_{n}\right)\left(u_{0}^{(n)}, \psi^{(n)}\right) \\
= & \mathcal{S}(t, t-T) \mathcal{S}\left(t-T, t-s_{n}\right)\left(u_{0}^{(n)}, \psi^{(n)}\right) \\
\rightarrow & \mathcal{S}(t, t-T)\left(w_{T}, \psi_{T}\right) \text { weakly in } W \times L_{W}^{2} \text { as } n \rightarrow \infty .
\end{aligned}
$$

By the uniqueness of the limit, we get $\left(w_{0}, \psi\right)=\mathcal{S}(t, t-T)\left(w_{T}, \psi_{T}\right)$ for each $T \in \mathbb{N}$. Setting

$$
\mathcal{S}\left(t-T, t-s_{n}\right)\left(u_{0}^{(n)}, \psi^{(n)}\right)=\left(w_{T}^{(n)}, \psi_{T}^{(n)}\right)
$$

we have

$$
\begin{aligned}
\mathcal{S}\left(t, t-s_{n}\right)\left(u_{0}^{(n)}, \psi^{(n)}\right) & =\mathcal{S}(t, t-T)\left(w_{T}^{(n)}, \psi_{T}^{(n)}\right) \\
& \rightarrow \mathcal{S}(t, t-T)\left(w_{T}, \psi_{T}\right) \\
& =\left(w_{0}, \psi\right) \text { weakly in } W \times L_{W}^{2} \text { as } n \rightarrow \infty .
\end{aligned}
$$

Denote by $u(t)=u\left(t ; \tau,\left(u_{0}, \psi\right)\right)$ the solution of problem (2.14) corresponding to the initial data $\left(u_{0}, \psi\right)$ and the initial time $\tau \in \mathbb{R}$. Write

$$
u^{(n)}(t)=u\left(t ; t-s_{n},\left(u_{0}^{(n)}, \psi^{(n)}\right)\right)=u\left(t ; t-T,\left(w_{T}^{(n)}, \psi_{T}^{(n)}\right)\right) .
$$

Then (4.78) implies that

$$
u^{(n)}(t)=u\left(t ; t-T,\left(w_{T}^{(n)}, \psi_{T}^{(n)}\right)\right) \rightarrow w_{0} \text { weakly in } W \text { as } n \rightarrow+\infty .
$$


Lemma 2.1 shows that the inner product $a(\cdot, \cdot)$ can induce a norm which is equivalent to $\|\cdot\|_{W}$. Thus (4.83) gives us

$$
a\left(w_{0}, w_{0}\right) \leq \liminf _{n} a\left(u\left(t ; t-T,\left(w_{T}^{(n)}, \psi_{T}^{(n)}\right)\right), u\left(t ; t-T,\left(w_{T}^{(n)}, \psi_{T}^{(n)}\right)\right)\right)
$$

We next prove

$$
a\left(w_{0}, w_{0}\right) \geq \limsup _{n} a\left(u\left(t ; t-T,\left(w_{T}^{(n)}, \psi_{T}^{(n)}\right)\right), u\left(t ; t-T,\left(w_{T}^{(n)}, \psi_{T}^{(n)}\right)\right)\right) .
$$

Define $\llbracket \cdot, \cdot \rrbracket: D(A) \times D(A) \longmapsto \mathbb{R}$ as

$$
\llbracket u, v \rrbracket=2 \mu_{1}(A u, A v)-\frac{c_{1}^{2}}{c_{2}} \mu_{1} a(u, v), \quad \forall u, v \in D(A) \cap W .
$$

Then we have, using (2.7) and (2.8),

$$
\begin{aligned}
\llbracket u \rrbracket^{2}=\llbracket u, u \rrbracket & \geq 2 \mu_{1}\|A u\|^{2}-c_{1}^{2} \mu_{1}\|u\|_{W}^{2} \\
& \geq 2 \mu_{1}\|A u\|^{2}-\mu_{1}\|A u\|^{2}=\mu_{1}\|A u\|^{2}
\end{aligned}
$$

and

$$
\mu_{1}\|A u\|^{2} \leq \llbracket u \rrbracket^{2} \leq 2 \mu_{1}\|A u\|^{2}
$$

Let $k=\frac{c_{1}^{2}}{c_{2}}$. Multiplying the equation in (2.14) by $A u(t)$, we obtain

$$
\begin{aligned}
& \frac{d}{d t} a(u(t), u(t))+k \mu_{1} a(u(t), u(t)) \\
& =\quad(g, A u(t))+\left(f\left(t, u_{t}\right), A u(t)\right) \\
& \quad-\langle B(u(t)), A u(t)\rangle-\langle N(u(t)), A u(t)\rangle-\llbracket u(t) \rrbracket^{2} .
\end{aligned}
$$

Using the formula of constant variation, we get

$$
a(u(t), u(t))=a\left(u_{0}, u_{0}\right) e^{-k \mu_{1}(t-\tau)}+\int_{\tau}^{t} e^{-k \mu_{1}(t-\xi)} \mathscr{K}\left(g, f\left(\xi, u_{\xi}\right), u(\xi)\right) d \xi
$$

where

$$
\begin{gathered}
\mathscr{K}\left(g, f\left(\xi, u_{\xi}\right), u(\xi)\right)=\quad(g, A u(\xi))+\left(f\left(\xi, u_{\xi}\right), A u(\xi)\right)-\langle B(u(\xi)), A u(\xi)\rangle \\
-\langle N(u(\xi)), A u(\xi)\rangle-\llbracket u(\xi) \rrbracket^{2} .
\end{gathered}
$$


Now by (4.82) and (4.88), we have

$$
\begin{aligned}
& a\left(u^{(n)}(t), u^{(n)}(t)\right) \\
= & a\left(w_{T}^{(n)}, \psi_{T}^{(n)}\right) e^{-k \mu_{1} T} \\
+ & \int_{t-T}^{t} e^{-k \mu_{1}(t-\xi)}\left(g, A u\left(\xi ; t-T,\left(w_{T}^{(n)}, \psi_{T}^{(n)}\right)\right)\right) d \xi \\
+ & \int_{t-T}^{t} e^{-k \mu_{1}(t-\xi)}\left(f\left(\xi, u_{\xi}\left(\cdot ; t-T,\left(w_{T}^{(n)}, \psi_{T}^{(n)}\right)\right)\right), A u\left(\xi ; t-T,\left(w_{T}^{(n)}, \psi_{T}^{(n)}\right)\right)\right) d \xi \\
- & \int_{t-T}^{t} e^{-k \mu_{1}(t-\xi)}\left\langle B\left(u\left(\xi ; t-T,\left(w_{T}^{(n)}, \psi_{T}^{(n)}\right)\right)\right), A u\left(\xi ; t-T,\left(w_{T}^{(n)}, \psi_{T}^{(n)}\right)\right)\right\rangle d \xi \\
- & \int_{t-T}^{t} e^{-k \mu_{1}(t-\xi)}\left\langle N\left(u\left(\xi ; t-T,\left(w_{T}^{(n)}, \psi_{T}^{(n)}\right)\right)\right), A u\left(\xi ; t-T,\left(w_{T}^{(n)}, \psi_{T}^{(n)}\right)\right)\right\rangle d \xi \\
- & \int_{t-T}^{t} e^{-k \mu_{1}(t-\xi)} \llbracket u\left(\xi ; t-T,\left(w_{T}^{(n)}, \psi_{T}^{(n)}\right)\right) \rrbracket^{2} d \xi .
\end{aligned}
$$

We next evaluate the terms in the right-hand side of (4.89). Firstly, by Lemma 3.6, we have

$$
a\left(w_{T}^{(n)}, \psi_{T}^{(n)}\right) e^{-k \mu_{1} T} \leq c_{2}^{2}\left\|w_{T}^{(n)}\right\|_{W}^{2} e^{-k \mu_{1} T} \leq c_{2}^{2} R_{W}^{2} e^{-k \mu_{1} T},
$$

when $n$ is large enough such that $s_{n}>T+T^{*}$ (see (4.77)). Secondly, the following three limiting relations can be proved essentially by the same way as that in [56]:

$$
\begin{aligned}
& \int_{t-T}^{t} e^{-k \mu_{1}(t-\xi)} \llbracket u\left(t ; t-T,\left(w_{T}^{(n)}, \psi_{T}^{(n)}\right)\right) \rrbracket^{2} d \xi \\
\quad \leq \liminf _{n} \int_{t-T}^{t} e^{-k \mu_{1}(t-\xi)} \llbracket u\left(t ; t-T,\left(w_{T}, \psi_{T}\right) \rrbracket^{2} d \xi\right. & \lim _{n \rightarrow \infty} \int_{t-T}^{t} e^{-k \mu_{1}(t-\xi)}\left\langle B\left(u\left(\xi ; t-T,\left(w_{T}^{(n)}, \psi_{T}^{(n)}\right)\right)\right), A u\left(\xi ; t-T,\left(w_{T}^{(n)}, \psi_{T}^{(n)}\right)\right)\right\rangle d \xi \\
= & \int_{t-T}^{t} e^{-k \mu_{1}(t-\xi)}\left\langle B\left(u\left(\xi ; t-T,\left(w_{T}, \psi_{T}\right)\right)\right), A u\left(\xi ; t-T,\left(w_{T}, \psi_{T}\right)\right)\right\rangle d \xi \\
& \lim _{n \rightarrow \infty} \int_{t-T}^{t} e^{-k \mu_{1}(t-\xi)}\left\langle N\left(u\left(\xi ; t-T,\left(w_{T}^{(n)}, \psi_{T}^{(n)}\right)\right)\right), A u\left(\xi ; t-T,\left(w_{T}^{(n)}, \psi_{T}^{(n)}\right)\right)\right\rangle d \xi \\
= & \int_{t-T}^{t} e^{-k \mu_{1}(t-\xi)}\left\langle N\left(u\left(\xi ; t-T,\left(w_{T}, \psi_{T}\right)\right)\right), A u\left(\xi ; t-T,\left(w_{T}, \psi_{T}\right)\right)\right\rangle d \xi .
\end{aligned}
$$

Finally, we prove

$$
\begin{aligned}
& \lim _{n \rightarrow \infty} \int_{t-T}^{t} e^{-k \mu_{1}(t-\xi)}\left(f\left(\xi, u_{\xi}\left(\cdot ; t-T,\left(w_{T}^{(n)}, \psi_{T}^{(n)}\right)\right)\right), A u\left(\xi ; t-T,\left(w_{T}^{(n)}, \psi_{T}^{(n)}\right)\right)\right) d \xi \\
= & \int_{t-T}^{t} e^{-k \mu_{1}(t-\xi)}\left(f\left(\xi, u_{\xi}\left(\cdot ; t-T,\left(w_{T}, \psi_{T}\right)\right)\right), A u\left(\xi ; t-T,\left(w_{T}, \psi_{T}\right)\right)\right) d \xi .
\end{aligned}
$$

On the one hand, the embedding $W \times L_{W}^{2} \hookrightarrow H \times L_{H}^{2}$ is compact. Thus, by (4.79), we have

$$
\left(w_{T}^{(n)}, \psi_{T}^{(n)}\right) \longrightarrow\left(w_{T}, \psi_{T}\right) \text { strongly in } H \times L_{H}^{2} \text { as } n \rightarrow \infty \text {. }
$$


Then by Lemma 3.1 (II),

$$
\lim _{n \rightarrow \infty}\left\|u_{\xi}\left(\cdot ; t-T,\left(w_{T}^{(n)}, \psi_{T}^{(n)}\right)\right)-u_{\xi}\left(\cdot ; t-T,\left(w_{T}, \psi_{T}\right)\right)\right\|_{\mathcal{C}_{H}}=0,
$$

and by $\left(\mathbf{A}_{3}\right)$,

$$
\lim _{n \rightarrow \infty}\left\|f\left(\xi, u_{\xi}\left(\cdot ; t-T,\left(w_{T}^{(n)}, \psi_{T}^{(n)}\right)\right)\right)-f\left(\xi, u_{\xi}\left(\cdot ; t-T,\left(w_{T}, \psi_{T}\right)\right)\right)\right\|=0 .
$$

At the same time, from Theorem $2.1(\mathbf{2})$, we see that for each $n \in \mathbb{N}$,

$$
\int_{t-T}^{t}\left\|A u_{\xi}\left(\cdot ; t-T,\left(w_{T}^{(n)}, \psi_{T}^{(n)}\right)\right)\right\|^{2} d \xi<+\infty .
$$

It then follows from (4.97) and (4.98) that

$$
\begin{aligned}
& \mid \int_{t-T}^{t} e^{-k \mu_{1}(t-\xi)}\left(f\left(\xi, u_{\xi}\left(\cdot ; t-T,\left(w_{T}^{(n)}, \psi_{T}^{(n)}\right)\right)\right), A u\left(\xi ; t-T,\left(w_{T}^{(n)}, \psi_{T}^{(n)}\right)\right)\right) d \xi \\
& -\int_{t-T}^{t} e^{-k \mu_{1}(t-\xi)}\left(f\left(\xi, u_{\xi}\left(\cdot ; t-T,\left(w_{T}, \psi_{T}\right)\right)\right), A u\left(\xi ; t-T,\left(w_{T}^{(n)}, \psi_{T}^{(n)}\right)\right)\right) d \xi \mid \\
\leq \quad & \left\{\int_{t-T}^{t} e^{-k \mu_{1}(t-\xi)} \| f\left(\xi, u_{\xi}\left(\cdot ; t-T,\left(w_{T}^{(n)}, \psi_{T}^{(n)}\right)\right)\right)\right. \\
& \left.-f\left(\xi, u_{\xi}\left(\cdot ; t-T,\left(w_{T}, \psi_{T}\right)\right)\right) \|^{2} d \xi\right\}^{1 / 2} \\
& \cdot\left(\int_{t-T}^{t} e^{-k \mu_{1}(t-\xi)}\left\|A u\left(\xi ; t-T,\left(w_{T}^{(n)}, \psi_{T}^{(n)}\right)\right)\right\|^{2} d \xi\right)^{1 / 2} \\
\longrightarrow & 0 \text { as } n \rightarrow \infty .
\end{aligned}
$$

On the other hand, since $\left(w_{T}^{(n)}, \psi_{T}^{(n)}\right) \rightarrow\left(w_{T}, \psi_{T}\right)$ weakly in $W \times L_{W}^{2}$ as $n \rightarrow \infty$, we can use the similar argument as that in [56 to prove

$u\left(\xi ; t-T,\left(w_{T}^{(n)}, \psi_{T}^{(n)}\right)\right) \rightarrow u\left(\xi ; t-T,\left(w_{T}, \psi_{T}\right)\right)$ weakly in $L^{2}(t-T, t ; D(A))$ as $n \rightarrow \infty$.

Thus,

$$
\begin{array}{r}
A u\left(\xi ; t-T,\left(w_{T}^{(n)}, \psi_{T}^{(n)}\right)\right) \rightarrow A u\left(\xi ; t-T,\left(w_{T}, \psi_{T}\right)\right) \\
\text { weakly in } L^{2}(t-T, t ; H) \text { as } n \rightarrow \infty .
\end{array}
$$

Obviously, $e^{-k \mu_{1}(t-\xi)} f\left(\xi, u_{\xi}\left(\cdot ; t-T,\left(w_{T}, \psi_{T}\right)\right)\right) \in L^{2}(t-T, t ; H)$ and then by (4.100),

$$
\begin{aligned}
& \mid \int_{t-T}^{t} e^{-k \mu_{1}(t-\xi)}\left(f\left(\xi, u_{\xi}\left(\cdot ; t-T,\left(w_{T}, \psi_{T}\right)\right)\right), A u\left(\xi ; t-T,\left(w_{T}^{(n)}, \psi_{T}^{(n)}\right)\right)\right) d \xi \\
& -\int_{t-T}^{t} e^{-k \mu_{1}(t-\xi)}\left(f\left(\xi, u_{\xi}\left(\cdot ; t-T,\left(w_{T}, \psi_{T}\right)\right)\right), A u\left(\xi ; t-T,\left(w_{T}, \psi_{T}\right)\right)\right) d \xi \mid \\
& \longrightarrow 0 \text { as } n \rightarrow \infty .
\end{aligned}
$$


(4.99) and (4.101) imply (4.94). Now taking (4.90)-(4.94) into account, we get

$$
\begin{aligned}
& \limsup _{n \rightarrow \infty} a\left(u^{(n)}(t), u^{(n)}(t)\right) \\
\leq & c_{2}^{2} R_{W}^{2} e^{-k \mu_{1} T}+\int_{t-T}^{t} e^{-k \mu_{1}(t-\xi)}\left(g, A u\left(\xi ; t-T,\left(w_{T}, \psi_{T}\right)\right)\right) d \xi \\
& +\int_{t-T}^{t} e^{-k \mu_{1}(t-\xi)}\left(f\left(\xi, u_{\xi}\left(\cdot ; t-T,\left(w_{T}, \psi_{T}\right)\right)\right), A u\left(\xi ; t-T,\left(w_{T}, \psi_{T}\right)\right)\right) d \xi \\
& -\int_{t-T}^{t} e^{-k \mu_{1}(t-\xi)}\left\langle B\left(u\left(\xi ; t-T,\left(w_{T}, \psi_{T}\right)\right)\right), A u\left(\xi ; t-T,\left(w_{T}, \psi_{T}\right)\right)\right\rangle d \xi \\
& -\int_{t-T}^{t} e^{-k \mu_{1}(t-\xi)}\left\langle N\left(u\left(\xi ; t-T,\left(w_{T}, \psi_{T}\right)\right)\right), A u\left(\xi ; t-T,\left(w_{T}, \psi_{T}\right)\right)\right\rangle d \xi \\
& -\int_{t-T}^{t} e^{-k \mu_{1}(t-\xi)} \llbracket u\left(\xi ; t-T,\left(w_{T}, \psi_{T}\right)\right) \rrbracket^{2} d \xi .
\end{aligned}
$$

At the same time, applying (4.88) to $w_{0}=u\left(t ; t-T,\left(w_{T}, \psi_{T}\right)\right)$, we obtain

$$
\begin{aligned}
& a\left(w_{0}, w_{0}\right)=a\left(w_{T}, w_{T}\right) e^{-k \mu_{1} T}+\int_{t-T}^{t} e^{-k \mu_{1}(t-\tau)}\left(g, A u\left(\xi ; t-T,\left(w_{T}, \psi_{T}\right)\right)\right) d \xi \\
& +\int_{t-T}^{t} e^{-k \mu_{1}(t-\xi)}\left(f\left(\xi, u_{\xi}\left(\cdot ; t-T,\left(w_{T}, \psi_{T}\right)\right)\right), A u\left(\xi ; t-T,\left(w_{T}, \psi_{T}\right)\right)\right) d \xi \\
& -\int_{t-T}^{t} e^{-k \mu_{1}(t-\xi)}\left\langle B\left(u\left(\xi ; t-T,\left(w_{T}, \psi_{T}\right)\right)\right), A u\left(\xi ; t-T,\left(w_{T}, \psi_{T}\right)\right)\right\rangle d \xi \\
& -\int_{t-T}^{t} e^{-k \mu_{1}(t-\xi)}\left\langle N\left(u\left(\xi ; t-T,\left(w_{T}, \psi_{T}\right)\right)\right), A u\left(\xi ; t-T,\left(w_{T}, \psi_{T}\right)\right)\right\rangle d \xi \\
& -\int_{t-T}^{t} e^{-k \mu_{1}(t-\xi)} \llbracket u\left(\xi ; t-T,\left(w_{T}, \psi_{T}\right)\right) \rrbracket^{2} d \xi, \quad \text { for each } T \in \mathbb{N} .
\end{aligned}
$$

From (4.102) and (4.103) we get that for each $T \in \mathbb{N}$,

$$
\limsup _{n \rightarrow \infty} a\left(u^{(n)}(t), u^{(n)}(t)\right) \leq\left(c_{2}^{2} R_{W}^{2}-a\left(w_{T}, w_{T}\right)\right) e^{-k \mu_{1} T}+a\left(w_{0}, w_{0}\right) .
$$

Recalling that $w_{T} \in \mathscr{B}_{W}\left(0, R_{W}\right)$, and letting $T \rightarrow+\infty$ in (4.104), we get

$$
\limsup _{n \rightarrow \infty} a\left(u^{(n)}(t), u^{(n)}(t)\right) \leq a\left(w_{0}, w_{0}\right),
$$

that is, (4.85) holds. It follows from (4.84) and (4.85) that

$$
\lim _{n \rightarrow \infty} a\left(u^{(n)}(t), u^{(n)}(t)\right)=\lim _{n \rightarrow \infty} a\left(u\left(t ; t-s_{n},\left(u_{0}^{(n)}, \psi^{(n)}\right)\right)\right)=a\left(w_{0}, w_{0}\right),
$$

and then Lemma 2.1 shows that

$$
\left.\lim _{n \rightarrow \infty} \| u^{(n)}(t)\right)\left\|_{W}=\right\| w_{0} \|_{W} .
$$

Since $W$ is a Hilbert space, combining (4.78) and (4.106), we have

$$
\left.\lim _{n \rightarrow \infty} \| u^{(n)}(t)\right)-w_{0} \|_{W}=0
$$

i.e.

$$
u^{(n)}(t) \longrightarrow w_{0} \quad \text { strongly in } W \text { as } n \rightarrow \infty
$$


Now we denote by $u_{t}\left(\theta ; t-s_{n},\left(u_{0}, \psi\right)\right)=u\left(t+\theta ; t-s_{n},\left(u_{0}, \psi\right)\right) \in L_{W}^{2}$ the solution of (2.14) with initial data $\left(u_{0}, \psi\right) \in L_{W}^{2}$ at initial time $t-s_{n}$. Then for each $\theta_{0} \in[-\nu, 0]$, we get

$$
u_{t}\left(\theta_{0} ; t-s_{n},\left(u_{0}^{(n)}, \psi^{(n)}\right)\right) \doteq u_{t}^{(n)}\left(\theta_{0}\right)=u\left(t+\theta_{0} ; t-s_{n},\left(u_{0}^{(n)}, \psi^{(n)}\right)\right) \longrightarrow \psi\left(\theta_{0}\right)
$$

strongly in $W$ as $n \rightarrow \infty$. Therefore, we have

$$
\left\|u_{t}^{(n)}(\theta)\right\|_{W}^{2} \longrightarrow\|\psi(\theta)\|_{W}^{2} \text { everywhere for } \theta \in[-\nu, 0] \text { as } n \rightarrow \infty .
$$

Note that $u_{t}^{(n)}\left(\theta_{0}\right) \in \mathscr{B}_{W}\left(0, R_{W}\right)$ for $n$ being large enough and $\psi(\theta) \in L_{W}^{2}$. By Lebesgue's Dominated Convergence Theorem, we obtain

$$
\lim _{n \rightarrow \infty} \int_{-\nu}^{0}\left\|u_{t}^{(n)}(\theta)\right\|_{W}^{2} d \theta=\int_{-\nu}^{0}\|\psi(\theta)\|_{W}^{2} d \theta
$$

By (4.78),

$$
u_{t}^{(n)}(\theta) \rightarrow \psi(\theta) \text { weakly in } L_{W}^{2} \text { as } n \rightarrow \infty .
$$

Also, $L_{W}^{2}$ is a Hilbert space, and (4.108) and (4.109) imply

$$
u_{t}^{(n)}(\theta) \longrightarrow \psi(\theta) \text { strongly in } L_{W}^{2} \text { as } n \rightarrow+\infty .
$$

So (4.107) and (4.110) tell us that

$$
\begin{aligned}
S\left(t, t-s_{n}\right)\left(u_{0}^{(n)}, \psi^{(n)}\right) & =\left(u\left(t ; t-s_{n},\left(u_{0}^{(n)}, \psi^{(n)}\right)\right), u_{t}\left(\theta ; t-s_{n},\left(u_{0}^{(n)}, \psi^{(n)}\right)\right)\right) \\
& \longrightarrow\left(w_{0}, \psi(\theta)\right) \text { strongly in } E_{W}^{2} \text { as } n \rightarrow \infty .
\end{aligned}
$$

The proof of Lemma 4.1 is now complete.

The main result of this section is

Theorem 4.1. Let $\left(\mathbf{A}_{\mathbf{1}}\right)-\left(\mathbf{A}_{\mathbf{5}}\right)$ hold and $g \in H$. Then the process $\{\mathcal{S}(t, \tau)\}_{t \geq \tau}$ possesses a unique uniformly bounded pullback attractor $\left\{\mathscr{A}_{E_{W}^{2}}(t)\right\}_{t \in \mathbb{R}} \subset \mathscr{B}_{W}\left(0, R_{W}\right) \times$ $\mathscr{B}_{L_{W}^{2}}\left(0, \nu^{1 / 2} R_{W}\right) \subset E_{W}^{2}$. Furthermore, $\mathscr{A}_{E_{W}^{2}}(t) \subset W \times \mathcal{C}_{W}$ for all $t \in \mathbb{R}$.

Proof. Lemma 3.6 shows that $\{\mathcal{S}(t, \tau)\}_{t \geq \tau}$ possesses a family of uniformly bounded pullback absorbing sets in $E_{W}^{2}$, while Lemma 4.1 shows that $\{\mathcal{S}(t, \tau)\}_{t \geq \tau}$ is pullback asymptotically compact in $E_{W}^{2}$. At the same time, the continuity of $\{\mathcal{S}(t, \tau)\}_{t \geq \tau}$ in $E_{W}^{2}$ is obvious. Thus, we can prove, similar to the semigroup case (see, e.g., [53]) that $\{\mathcal{S}(t, \tau)\}_{t \geq \tau}$ possesses a unique uniformly bounded pullback attractor $\left\{\mathscr{A}_{E_{W}^{2}}(t)\right\}_{t \in \mathbb{R}} \subset$ $\mathscr{B}_{W}\left(0, R_{W}\right) \times \mathscr{B}_{L_{W}^{2}}\left(0, \nu^{1 / 2} R_{W}\right) \subset E_{W}^{2}$. The fact that $\mathscr{A}_{E_{W}^{2}}(t) \subset W \times \mathcal{C}_{W}$ for all $t \in \mathbb{R}$ follows from Theorem 2.1 (2) and Lemma 3.6. The proof is complete.

Remark 4.1. Let $\mathcal{H}$ be a Hilbert space or a uniform convex Banach space. If $h_{n} \rightarrow h$ weakly in $\mathcal{H}$ and $\left\|h_{n}\right\|_{\mathcal{H}} \longrightarrow\|h\|_{\mathcal{H}}$ as $n \rightarrow \infty$, then $h_{n} \longrightarrow h$ strongly in $\mathcal{H}$. This property of a Hilbert space plays a very important role when we prove Lemma 4.1 via the energy equality method. Since $\mathcal{C}_{W}$ is not a Hilbert space, the energy equality method seems difficult to be applicable for us to prove that the process $\{\mathcal{U}(t, \tau)\}_{t \geq \tau}$ possesses a pullback attractor $\left\{\mathscr{A}_{\mathcal{C}_{W}}(t)\right\}_{t \in \mathbb{R}}$ in $\mathcal{C}_{W}$. But we can prove that the pullback attractor $\left\{\mathscr{A}_{\mathcal{C}_{H}}(t)\right\}_{t \in \mathbb{R}}$ for $\{\mathcal{U}(t, \tau)\}_{t \geq \tau}$ in $\mathcal{C}_{H}$ is indeed a family of compact sets of $\mathcal{C}_{W}$ (see Lemma 5.2 below). 
5. Regularity of pullback attractors. In this section we first prove that the pullback attractor $\left\{\mathscr{A}_{E_{W}^{2}}(t)\right\}_{t \in \mathbb{R}}$ coincides with $\left\{\mathscr{A}_{E_{H}^{2}}(t)\right\}_{t \in \mathbb{R}}$. Then we establish that the process $\{\mathcal{U}(t, \tau)\}_{t \geq \tau}$ possesses a uniformly bounded pullback attractor $\left\{\mathscr{A}_{\mathcal{C}_{W}}(t)\right\}_{t \in \mathbb{R}}$ in $\mathcal{C}_{W}$, and moreover

$$
\mathscr{A}_{\mathcal{C}_{W}}(t)=\mathscr{A}_{\mathcal{C}_{H}}(t), \quad \forall t \in \mathbb{R} .
$$

We next use the Uniform Gronwall Lemma to prove that the solution $\mathcal{S}(t, t-s)\left(u_{0}, \psi\right)$ with initial condition $\left(u_{0}, \psi\right)$ in any bounded sets of $E_{H}^{2}$ will enter a bounded set of $E_{W}^{2}$ provided $s$ is large enough.

Lemma 5.1. Assume $\left(\mathbf{A}_{\mathbf{1}}\right)-\left(\mathbf{A}_{\mathbf{5}}\right)$ hold and $g \in H$. Let $\mathscr{B}_{E_{H}^{2}}$ be a bounded set of $E_{H}^{2}$. Then for any $\left(u_{0}, \psi\right) \in \mathscr{B}_{E_{H}^{2}}$ and any $t \in \mathbb{R}$, there exist a time $s_{0}^{*}=s_{0}^{*}\left(\mathscr{B}_{E_{H}^{2}}\right)$ and a positive constant $M$ such that

$$
\begin{aligned}
\left\|\mathcal{S}(t, t-s)\left(u_{0}, \psi\right)\right\|_{E_{W}^{2}} & =\|\left(u\left(t ; t-s,\left(u_{0}, \psi\right)\right), u_{t}\left(\cdot ; t-s,\left(u_{0}, \psi\right)\right) \|_{E_{W}^{2}}\right. \\
& \leq M, \quad \forall s \geq s_{0}^{*} .
\end{aligned}
$$

Proof. For simplicity, we denote $u^{\prime}=\frac{\partial u}{\partial t}$ and $u=u(t)=u\left(t ; t-s,\left(u_{0}, \psi\right)\right)$. Multiplying the equation in (2.14) by $u^{\prime}$ and then integrating the resulting equality over $\Omega$, we obtain

$$
\left\|u^{\prime}\right\|^{2}+2 \mu_{1} a\left(u, u^{\prime}\right)+\left\langle B(u), u^{\prime}\right\rangle+\left\langle N(u), u^{\prime}\right\rangle=\left(g, u^{\prime}\right)+\left(f\left(t, u_{t}\right), u^{\prime}\right) .
$$

Set

Then

$$
\Gamma\left(|e(u)|^{2}\right)=\int_{0}^{|e(u)|^{2}} \mu_{0}(\varepsilon+\xi)^{-\alpha / 2} d \xi
$$

$$
\frac{d \Gamma}{d t}=\sum_{i, j=1}^{2} \mu(u) e_{i j}(u) \frac{\partial e_{i j}(u)}{\partial t}=\sum_{i, j=1}^{2} \mu(u) e_{i j}(u) e_{i j}\left(u^{\prime}\right) .
$$

Thus,

$$
\left\langle N(u), u^{\prime}\right\rangle=\sum_{i, j=1}^{2} \int_{\Omega} \mu(u) e_{i j}(u) e_{i j}\left(u^{\prime}\right) d x=\frac{d}{d t}\left(\int_{\Omega} \Gamma\left(|e(u)|^{2}\right) d x\right) .
$$

Substituting (5.113) into (5.112), we obtain

$$
\begin{aligned}
& \left\|u^{\prime}\right\|^{2}+\frac{d}{d t}\left(\mu_{1} a(u, u)+\int_{\Omega} \Gamma\left(|e(u)|^{2}\right) d x\right) \\
= & -\left\langle B(u), u^{\prime}\right\rangle+\left(g, u^{\prime}\right)+\left(f\left(t, u_{t}\right), u^{\prime}\right) \\
\leq & \left|\sum_{i, j=1}^{2} \int_{\Omega} u_{i} \frac{\partial u_{j}}{\partial x_{i}} u_{j}^{\prime} d x\right|+\|g\|^{2}+\left\|f\left(t, u_{t}\right)\right\|^{2}+\frac{1}{2}\left\|u^{\prime}\right\|^{2} \\
\leq & \|u\|_{L^{4}(\Omega)}\|\nabla u\|_{L^{4}(\Omega)}\left\|u^{\prime}\right\|+\|g\|^{2}+\left\|f\left(t, u_{t}\right)\right\|^{2}+\frac{1}{2}\left\|u^{\prime}\right\|^{2} .
\end{aligned}
$$

By the Gagliardo-Nirenberg inequality,

$$
\|u\|_{L^{4}(\Omega)}\|\nabla u\|_{L^{4}(\Omega)}\left\|u^{\prime}\right\| \leq c\|\Delta u\|^{2}\left\|u^{\prime}\right\| \leq \frac{c^{2}}{2}\|u\|_{W}^{4}+\frac{1}{2}\left\|u^{\prime}\right\|^{2} .
$$


Inserting (5.115) into (5.114), we obtain by using Lemma 2.1 that

$$
\begin{aligned}
\frac{d}{d t}\left(\mu_{1} a(u, u)+\int_{\Omega} \Gamma\left(|e(u)|^{2}\right) d x\right) & \leq \frac{c^{2}}{2}\|u\|_{W}^{4}+\|g\|^{2}+\left\|f\left(t, u_{t}\right)\right\|^{2} \\
& \leq \frac{c^{2}}{2}\|u\|_{W}^{2} \cdot \frac{\mu_{1} a(u, u)}{c_{1} \mu_{1}}+\|g\|^{2}+\left\|f\left(t, u_{t}\right)\right\|^{2} .
\end{aligned}
$$

So we have

$$
\frac{d \Phi(t)}{d t} \leq \Upsilon(t) \Phi(t)+\Psi(t)
$$

where

$$
\begin{aligned}
& \Phi(t)=\mu_{1} a(u(t), u(t))+\int_{\Omega} \Gamma\left(|e(u(t))|^{2}\right) d x, \\
& \Upsilon(t)=\frac{c^{2}}{2 c_{1} \mu_{1}}\|u(t)\|_{W}^{2}, \quad \Psi(t)=\|g\|^{2}+\left\|f\left(t, u_{t}\right)\right\|^{2} .
\end{aligned}
$$

Now taking the inner product $(\cdot, \cdot)$ of the equation in (2.14) with $u$ and integrating the resulting equality over $[t, t+1]$, we obtain

$$
\begin{aligned}
& \int_{t}^{t+1} 2 \mu_{1} a(u(\xi), u(\xi)) d \xi \\
\leq & \int_{t}^{t+1}\|u(\xi)\|\left(\|g(\xi)\|+\left\|f\left(\xi, u_{\xi}\right)\right\|\right) d \xi+\frac{1}{2}\|u(t)\|^{2} \\
\leq & \int_{t}^{t+1}\left(\|u(\xi)\|^{2}+2\|g(\xi)\|^{2}+2\left\|f\left(\xi, u_{\xi}\right)\right\|^{2}\right) d \xi+\frac{1}{2}\|u(t)\|^{2} .
\end{aligned}
$$

Denote by $s_{0}\left(\mathscr{B}_{E_{H}^{2}}\right)$ the absorbing time corresponding to $\mathscr{B}_{H}\left(0, R_{H}\right) \times \mathscr{B}_{L_{H}^{2}}\left(0, \nu^{1 / 2} R_{H}\right)$ in Lemma 3.4 and let $s \geq s_{0}$. Then from (5.118) it follows that

$$
\begin{aligned}
2 c_{1} \mu_{1} \int_{t}^{t+1}\|u(\xi)\|_{W}^{2} d \xi & \leq \int_{t}^{t+1} 2 \mu_{1} a(u(\xi), u(\xi)) d \xi \\
& \leq R_{H}^{2}+2\|g\|^{2}+2 \int_{t}^{t+1}\left\|f\left(\xi, u_{\xi}\right)\right\|^{2} d \xi+\frac{1}{2} R_{H}^{2} \\
& \leq \frac{3}{2} R_{H}^{2}+2\|g\|^{2}+2 C_{f}^{2} \int_{t}^{t+1}\left\|u_{\xi}\right\|_{\mathcal{C}_{H}}^{2} d \xi \\
& \leq \frac{3}{2} R_{H}^{2}+2\|g\|^{2}+2 C_{f}^{2} R_{H}^{2}, \quad \forall s \geq s_{0} .
\end{aligned}
$$

Hence, by Lemma 2.1,

$$
\begin{aligned}
\int_{t}^{t+1} \Upsilon(\xi) d \xi & =\int_{t}^{t+1} \frac{c^{2}}{2 c_{1} \mu_{1}}\|u(\xi)\|_{W}^{2} d \xi \leq \frac{c^{2}}{4 c_{1}^{2} \mu_{1}^{2}}\left(\frac{3}{2} R_{H}^{2}+2\|g\|^{2}+2 C_{f}^{2} R_{H}^{2}\right) \\
& \doteq a_{1}, \quad \forall s \geq s_{0} .
\end{aligned}
$$

Clearly, we have

$$
\int_{t}^{t+1} \Psi(\xi) d \xi=\int_{t}^{t+1}\left(\|g\|^{2}+\left\|f\left(\xi, u_{\xi}\right)\right\|^{2}\right) d \xi \leq\|g\|^{2}+C_{f}^{2} R_{H}^{2} \doteq a_{2} . \quad \forall s \geq s_{0},
$$


where $a_{1}, a_{2}$ are positive constants. We next show that there exists a positive constant $a_{3}$ such that

$$
\int_{t}^{t+1} \Phi(\xi) d \xi \leq a_{3}, \quad \forall s \geq s_{0} .
$$

From (5.119) and Lemma 2.1 we obtain

$$
\int_{t}^{t+1} \mu_{1} a(u(\xi), u(\xi)) d \xi \leq \frac{3}{4} R_{H}^{2}+\|g\|^{2}+C_{f}^{2} R_{H}^{2}, \quad \forall s \geq s_{0} .
$$

At the same time, we have $0<(\varepsilon+\xi)^{-\alpha / 2} \leq \varepsilon^{-\alpha / 2}$ for all $\xi \geq 0$ and $0<\alpha<1$. Thus

$$
\Gamma\left(|e(u)|^{2}\right)=\int_{0}^{|e(u)|^{2}} \mu_{0}(\varepsilon+\xi)^{-\alpha / 2} d \xi \leq \mu_{0} \varepsilon^{-\alpha / 2}|e(u)|^{2},
$$

from which and (5.122) it follows that

$$
\begin{aligned}
\int_{t}^{t+1} \int_{\Omega} \Gamma\left(|e(u(\xi))|^{2}\right) d x d \xi & \leq \mu_{0} \varepsilon^{-\alpha / 2} \int_{t}^{t+1} \int_{\Omega}|e(u(\xi))|^{2} d x d \xi \\
& \leq 4 \mu_{0} \varepsilon^{-\alpha / 2} \int_{t}^{t+1}\|u(\xi)\|_{W}^{2} d \xi \\
& \leq 4 \mu_{0} \varepsilon^{-\alpha / 2} \int_{t}^{t+1} \frac{\mu_{1} a(u(\xi), u(\xi))}{c_{1} \mu_{1}} d \xi \\
& \leq \frac{4 \mu_{0} \varepsilon^{-\alpha / 2}}{c_{1} \mu_{1}} \cdot\left(\frac{3}{4} R_{H}^{2}+\|g\|^{2}+C_{f}^{2} R_{H}^{2}\right), \forall s \geq s_{0},
\end{aligned}
$$

where we have used the fact that $\int_{\Omega}|e(u)|^{2} d x \leq 4\|u\|_{W}^{2}$. It follows from (5.122) and (5.123) that

$$
\int_{t}^{t+1} \Phi(\xi) d \xi \leq\left(1+\frac{4 \mu_{0} \varepsilon^{-\alpha / 2}}{c_{1} \mu_{1}}\right)\left(\frac{3}{4} R_{H}^{2}+\|g\|^{2}+C_{f}^{2} R_{H}^{2}\right) \doteq a_{3}, \quad \forall s \geq s_{0} .
$$

Taking Lemma 2.3, (5.117), (5.120), (5.121) and (5.124) into account, we obtain

$$
\Phi(t) \leq\left(a_{3}+a_{2}\right) e^{a_{1}}, \quad \forall s \geq s_{0}+1, \quad \forall t \in \mathbb{R} .
$$

Therefore,

$$
\|u(t)\|_{W}^{2} \leq \frac{1}{c_{1}} a(u(t), u(t)) \leq \frac{\Phi(t)}{c_{1} \mu_{1}} \leq \frac{\left(a_{3}+a_{2}\right) e^{a_{1}}}{c_{1} \mu_{1}} \doteq M_{3}, \quad s \geq s_{0}+1, \quad \forall t \in \mathbb{R},
$$

and if $s \geq s_{0}^{*}=s_{0}+\nu+1$, then

$$
\begin{aligned}
\left\|u_{t}\left(\cdot ; t-s,\left(u_{0}, \psi\right)\right)\right\|_{L_{W}^{2}}^{2} & =\int_{-\nu}^{0}\left\|u\left(t+\theta ; t-s,\left(u_{0}, \psi\right)\right)\right\|_{W}^{2} d \theta \\
& \leq \int_{-\nu}^{0} \sup _{\theta \in[-\nu, 0]}\left\|u\left(t+\theta ; t-s,\left(u_{0}, \psi\right)\right)\right\|_{W}^{2} d \theta \\
& \leq \nu M_{3}, \quad \forall t \in \mathbb{R}, \quad \forall s \geq s_{0}^{*} .
\end{aligned}
$$

The proof of Lemma 5.1 is now complete.

Theorem 5.1. Assume $\left(\mathbf{A}_{\mathbf{1}}\right)-\left(\mathbf{A}_{\mathbf{5}}\right)$ hold and $g \in H$. Then

$$
J\left(\mathscr{A}_{\mathcal{C}_{H}}(t)\right)=\mathscr{A}_{E_{H}^{2}}(t)=\mathscr{A}_{E_{W}^{2}}(t), \quad \forall t \in \mathbb{R} .
$$


Proof. By (3.70), we only need to prove

$$
\mathscr{A}_{E_{H}^{2}}(t)=\mathscr{A}_{E_{W}^{2}}(t), \quad \forall t \in \mathbb{R} .
$$

On the one hand, $\mathscr{A}_{E_{W}^{2}}(t)$ is bounded in $W \times L_{W}^{2}$ for any $t \in \mathbb{R}$. Thus $\mathscr{A}_{E_{W}^{2}}(t)$ is bounded in $H \times L_{H}^{2}$ for any $t \in \mathbb{R}$. By the invariance property and pullback attracting property of the pullback attractor, we have

$$
\begin{aligned}
\operatorname{Dist}_{E_{H}^{2}}\left(\mathscr{A}_{E_{W}^{2}}(t), \mathscr{A}_{E_{H}^{2}}(t)\right) & =\operatorname{Dist}_{E_{H}^{2}}\left(\mathcal{S}(t, t-s) \mathscr{A}_{E_{W}^{2}}(t-s), \mathscr{A}_{E_{H}^{2}}(t)\right) \quad\left(\forall s \in \mathbb{R}_{+}\right) \\
& =\lim _{s \rightarrow+\infty} \operatorname{Dist}_{E_{H}^{2}}\left(\mathcal{S}(t, t-s) \mathscr{A}_{E_{W}^{2}}(t-s), \mathscr{A}_{E_{H}^{2}}(t)\right) \\
& =0, \quad \forall t \in \mathbb{R},
\end{aligned}
$$

which implies

$$
\mathscr{A}_{E_{W}^{2}}(t) \subseteq \mathscr{A}_{E_{H}^{2}}(t), \quad \forall t \in \mathbb{R} .
$$

On the other hand, Theorem 4.1 and Lemma 5.1 show that $\mathscr{A}_{E_{H}^{2}}(t)$ is bounded in $W \times L_{W}^{2}$ for any $t \in \mathbb{R}$. Then also by the invariance property and pullback attracting property of the pullback attractor, we obtain

$$
\begin{aligned}
\operatorname{Dist}_{E_{H}^{2}}\left(\mathscr{A}_{E_{H}^{2}}(t), \mathscr{A}_{E_{W}^{2}}(t)\right) & \leq \operatorname{Dist}_{E_{W}^{2}}\left(\mathscr{A}_{E_{H}^{2}}(t), \mathscr{A}_{E_{W}^{2}}(t)\right) \\
& =\operatorname{Dist}_{E_{W}^{2}}\left(\mathcal{S}(t, t-s) \mathscr{A}_{E_{H}^{2}}(t-s), \mathscr{A}_{E_{W}^{2}}(t)\right)\left(\forall s \in \mathbb{R}_{+}\right) \\
& =\lim _{s \rightarrow+\infty} \operatorname{Dist}_{E_{W}^{2}}\left(\mathcal{S}(t, t-s) \mathscr{A}_{E_{H}^{2}}(t-s), \mathscr{A}_{E_{W}^{2}}(t)\right) \\
& =0, \quad \forall t \in \mathbb{R},
\end{aligned}
$$

which gives

$$
\mathscr{A}_{E_{H}^{2}}(t) \subseteq \mathscr{A}_{E_{W}^{2}}(t), \quad \forall t \in \mathbb{R} .
$$

We readily get (5.126) from (5.127) and (5.128). The proof is complete.

We next use (5.125) to prove that the pullback attractor $\left\{\mathscr{A}_{\mathcal{C}_{H}}(t)\right\}_{t \in \mathbb{R}}$ in $\mathcal{C}_{H}$ obtained in Theorem 3.1 is indeed a family of compact sets of $\mathcal{C}_{W}$.

Lemma 5.2. Assume $\left(\mathbf{A}_{\mathbf{1}}\right)-\left(\mathbf{A}_{\mathbf{5}}\right)$ hold and $g \in H$. Then the uniformly bounded pullback attractor $\left\{\mathscr{A}_{\mathcal{C}_{H}}(t)\right\}_{t \in \mathbb{R}}$ for $\{\mathcal{U}(t, \tau)\}_{t \geq \tau}$ in $\mathcal{C}_{H}$ is a family of compact sets of $\mathcal{C}_{W}$.

Proof. Given $t \in \mathbb{R}$, let $\left\{\psi_{n}(\theta)\right\}$ be a bounded sequence of $\mathscr{A}_{\mathcal{C}_{H}}(t)$. We only need to prove that $\left\{\psi_{n}(\theta)\right\}$ possesses a convergent subsequence in $\mathcal{C}_{W}$. Indeed, by (5.125) and Theorem 4.1 we see that $\mathscr{A}_{\mathcal{C}_{H}}(t) \subset \mathcal{C}_{W}$ and is compact in $\mathcal{C}_{H} \cap L_{W}^{2}$. Thus, there exist a subsequence (still denoted by $\left.\left\{\psi_{n}(\theta)\right\}\right)$ and a $\psi(\theta) \subset \mathcal{C}_{H} \cap L_{W}^{2}$ such that

$$
\begin{gathered}
\psi_{n}(\theta) \longrightarrow \psi(\theta) \text { strongly in } L_{W}^{2} \text { as } n \rightarrow \infty, \\
\psi_{n}(\theta) \longrightarrow \psi(\theta) \text { strongly in } \mathcal{C}_{H} \text { as } n \rightarrow \infty .
\end{gathered}
$$

From (5.129), we get

$$
\left\{\begin{array}{l}
\text { For any } \epsilon>0, \text { there exists an } N_{0} \in \mathbb{N}, \text { such that } \\
\int_{-\nu}^{0}\left\|\psi_{n}(\theta)-\psi_{m}(\theta)\right\|_{W}^{2} d \theta<\epsilon \text { provided } n, m>N_{0} .
\end{array}\right.
$$


Note that the strong convergence of the sequence $\left\{\psi_{n}(\theta)\right\}$ in $\mathcal{C}_{W}$ is equivalent to its uniform convergence. Therefore, if $\left\{\psi_{n}(\theta)\right\}$ is not uniformly convergent in $\mathcal{C}_{W}$, then by the Cauchy Criteria on the convergence of a sequence of functions, there exists an $\epsilon_{0}>0$ such that for any $N \in \mathbb{N}$, there exist $n_{0}, m_{0}>N$ and $\theta_{0} \in[-\nu, 0]$, yielding

$$
\left\|\psi_{n_{0}}\left(\theta_{0}\right)-\psi_{m_{0}}\left(\theta_{0}\right)\right\|_{W} \geq \epsilon_{0} .
$$

Since $\psi_{n_{0}}(\theta), \psi_{m_{0}}(\theta) \in \mathscr{A}_{\mathcal{C}_{H}}(t) \subset \mathcal{C}_{W}$, there exists a neighborhood of $\theta_{0}$ (denoted by $\left(\theta_{0}-\delta, \theta_{0}+\delta\right)$ for some $\left.\delta>0\right)$ such that

$$
\left\|\psi_{n_{0}}(\theta)-\psi_{m_{0}}(\theta)\right\|_{W} \geq \frac{\epsilon_{0}}{2} \text { for all } \theta \in\left(\theta_{0}-\delta, \theta_{0}+\delta\right) \subset[-\nu, 0],
$$

and thus we have

$$
\int_{-\nu}^{0}\left\|\psi_{n_{0}}(\theta)-\psi_{m_{0}}(\theta)\right\|_{W}^{2} d \theta \geq \int_{\theta_{0}-\delta}^{\theta_{0}+\delta}\left\|\psi_{n_{0}}(\theta)-\psi_{m_{0}}(\theta)\right\|_{W}^{2} d \theta \geq \delta \epsilon_{0}
$$

which contradicts with (5.131). Thus, $\left\{\psi_{n}(\theta)\right\}$ is uniformly convergent in $\mathcal{C}_{W}$. By (5.130) and the uniqueness of the limit, we get

$$
\psi_{n}(\theta) \longrightarrow \psi(\theta) \text { strongly in } \mathcal{C}_{W} .
$$

The proof is complete.

Theorem 5.2. Assume $\left(\mathbf{A}_{\mathbf{1}}\right)-\left(\mathbf{A}_{\mathbf{5}}\right)$ hold and $g \in H$. Then the process $\{\mathcal{U}(t, \tau)\}_{t \geq \tau}$ possesses a uniformly bounded pullback attractor $\left\{\mathscr{A}_{\mathcal{C}_{W}}(t)\right\}_{t \in \mathbb{R}}$ in $\mathcal{C}_{W}$. Moreover,

$$
\mathscr{A}_{\mathcal{C}_{W}}(t)=\mathscr{A}_{\mathcal{C}_{H}}(t) \text { and } J\left(\mathscr{A}_{\mathcal{C}_{W}}(t)\right)=J\left(\mathscr{A}_{\mathcal{C}_{H}}(t)\right)=\mathscr{A}_{E_{H}^{2}}(t)=\mathscr{A}_{E_{W}^{2}}(t), \forall t \in \mathbb{R} \text {. }
$$

Proof. We first prove that the process $\{\mathcal{U}(t, \tau)\}_{t \geq \tau}$ possesses a uniformly bounded pullback attractor $\left\{\mathscr{A}_{\mathcal{C}_{W}}(t)\right\}_{t \in \mathbb{R}}$ in $\mathcal{C}_{W}$. To this end, we establish that the family of uniformly bounded absorbing sets $\left\{\mathscr{B}_{\mathcal{C}_{W}}(t)\right\}_{t \in \mathbb{R}}$ of $\{\mathcal{U}(t, \tau)\}_{t \geq \tau}$ (see Lemma 3.6) are compact sets of $\mathcal{C}_{W}$. Indeed, the embedding $\mathcal{C}_{W} \hookrightarrow \mathcal{C}_{H}$ is continuous and for all $t \in \mathbb{R}$, $\mathscr{B}_{\mathcal{C}_{W}}(t)=\mathscr{B}_{\mathcal{C}_{W}}\left(0, R_{W}\right)$ is a bounded set of $\mathcal{C}_{H}$. Obviously, there exists a time $s^{*}=$ $s\left(\mathscr{B}_{\mathcal{C}_{W}}\left(0, R_{W}\right)\right)>0$ such that

$$
\mathcal{U}(t, t-s) \mathscr{B}_{\mathcal{C}_{W}}\left(0, R_{W}\right) \subset \mathscr{B}_{\mathcal{C}_{W}}\left(0, R_{W}\right), \quad \forall t \in \mathbb{R}, \quad \forall s>s^{*} .
$$

Thus,

$$
\begin{aligned}
\operatorname{Dist}_{\mathcal{C}_{H}}\left(\mathscr{B}_{\mathcal{C}_{W}}(t), \mathscr{A}_{\mathcal{C}_{H}}(t)\right) & =\operatorname{Dist}_{\mathcal{C}_{H}}\left(\mathscr{B}_{\mathcal{C}_{W}}\left(0, R_{W}\right), \mathscr{A}_{\mathcal{C}_{H}}(t)\right) \\
& \leq \lim _{s \rightarrow+\infty} \operatorname{Dist}_{\mathcal{C}_{H}}\left(\mathcal{U}(t, t-s) \mathscr{B}_{\mathcal{C}_{W}}\left(0, R_{W}\right), \mathscr{A}_{\mathcal{C}_{H}}(t)\right)=0,
\end{aligned}
$$

and $\mathscr{B}_{\mathcal{C}_{W}}(t) \subseteq \mathscr{A}_{\mathcal{C}_{H}}(t)$ for each $t \in \mathbb{R}$, while Lemma 5.2 shows that $\mathscr{A}_{\mathcal{C}_{H}}(t)$ is compact in $\mathcal{C}_{W}$. Hence $\mathscr{B}_{\mathcal{C}_{W}}(t)$ is compact in $\mathcal{C}_{W}$ and the process $\{\mathcal{U}(t, \tau)\}_{t \geq \tau}$ possesses a uniformly bounded pullback attractor $\left\{\mathscr{A}_{\mathcal{C}_{W}}(t)\right\}_{t \in \mathbb{R}}$ in $\mathcal{C}_{W}$. Recall that the embedding $\mathcal{C}_{W} \hookrightarrow$ $\mathcal{C}_{H}$ is continuous, so $\left\{\mathscr{A}_{\mathcal{C}_{W}}(t)\right\}_{t \in \mathbb{R}}$ is also a uniformly bounded pullback attractor for $\{\mathcal{U}(t, \tau)\}_{t \geq \tau}$ in $\mathcal{C}_{H}$. By the uniqueness of the (uniformly bounded) pullback attractor (see Remark 2.3), we get $\mathscr{A}_{\mathcal{C}_{W}}(t)=\mathscr{A}_{\mathcal{C}_{H}}(t)$ for each $t \in \mathbb{R}$, and then (5.133) is clear. The proof is complete. 
6. Conclusions and final remarks. We first use the idea of Caraballo and Real [19] to prove the existence of pullback attractors $\left\{\mathscr{A}_{\mathcal{C}_{H}}(t)\right\}_{t \in \mathbb{R}}$ and $\left\{\mathscr{A}_{E_{H}^{2}}(t)\right\}_{t \in \mathbb{R}}$ for the processes $\{\mathcal{U}(t, \tau)\}_{t \geq \tau}$ and $\{\mathcal{S}(t, \tau)\}_{t \geq \tau}$ in $\mathcal{C}_{H}$ and $E_{H}^{2}$, respectively. Then we apply the energy equality method of Ball to establish that the process $\{\mathcal{S}(t, \tau)\}_{t \geq \tau}$ is pullback asymptotically compact in $E_{W}^{2}$ and consequently, we obtain the existence of the uniformly bounded pullback attractor $\left\{\mathscr{A}_{E_{W}^{2}}(t)\right\}_{t \in \mathbb{R}}$ for $\{\mathcal{S}(t, \tau)\}_{t \geq \tau}$ in $E_{W}^{2}$. Meanwhile, we utilize the Uniform Gronwall Lemma to verify the regularity of the pullback attractor by showing $\mathscr{A}_{E_{W}^{2}}(t)=\mathscr{A}_{E_{H}^{2}}(t)$ for all $t \in \mathbb{R}$, which in turn allows us to prove the existence of a uniformly bounded pullback attractor $\left\{\mathscr{A}_{\mathcal{C}_{W}}(t)\right\}_{t \in \mathbb{R}}$ for $\{\mathcal{U}(t, \tau)\}_{t \geq \tau}$ in $\mathcal{C}_{W}$.

The regularity

$$
\mathscr{A}_{E_{W}^{2}}(t)=\mathscr{A}_{E_{H}^{2}}(t), \quad \mathscr{A}_{\mathcal{C}_{W}}(t)=\mathscr{A}_{\mathcal{C}_{H}}(t), \quad \text { for all } t \in \mathbb{R},
$$

shows that the pullback attractors associated to (1.1)-(1.2) do not depend on the energy space chosen for the mathematical studying. Furthermore, it reveals the pullback asymptotic smoothing effect of the fluid with delays in the sense that the solutions become eventually more regular (possessing $\mathrm{H}^{2}$-regularity) than the initial data (possessing $L^{2}$-regularity). This effect is caused essentially by the special extra stress tensor in the addressed equations of the non-Newtonian fluid. Also, this effect implies, in the case of delays, that the regularity of the fluid in its history state does not play an important role on the regularity of its eventual state.

We next give some remarks on some possible extensions.

REMARK 6.1. Caraballo and Real [19] gave some applications with forcing terms containing variable delays and distributed delays to the Navier-Stokes models. We find that these examples are also valid to the non-Newtonian fluid after some slight modifications. Similar to [19] for the Navier-Stokes models, some results for the autonomous version of the non-Newtonian fluid can be deduced as particular cases of the non-autonomous case discussed in this paper.

REMARK 6.2. If the spatial domain is unbounded, the embedding $W \hookrightarrow H$ is no longer compact and the argument used in the proof of Theorem 3.1 is inadequate. Caraballo, Łukaszewicz and Real [14] obtained the existence of pullback attractors for 2D nonautonomous Navier-Stokes equations in 2D unbounded domains in which the Poincaré inequality holds. The technique used in 14 is asymptotic compactness based on the energy equations of the associated systems. As mentioned previously in the introduction, this method was first formulated by Ball (see, e.g., [5) for autonomous systems, and by Lukaszewicz and Sadowski [40] to extend to the non-autonomous systems in the autonomous framework (see Ju [31] and Rosa [49]). This approach is also valid for the non-Newtonian fluid with delays when the spatial domain is unbounded but in which the Poincaré inequality holds.

REMARK 6.3. Zhao and Zhou [59] proved the existence and regularity of the pullback attractors (in the version of cocycle attractor) for the non-Newtonian fluid without delays. Schmalfuss [51] gave conditions for the existence of a pullback attractors for cocycles based on the so-called pullback convergence and proved the unique existence of pullback (or cocycle) attractor for the generalized non-autonomous Navier-Stokes equations. One can find that the conditions with respect to the $t$-regularity for the right-hand side in the 
addressed Navier-Stokes equations is weaker than that of [59]. It seems that we can also prove, using the argument of [51, the existence and regularity of cocycle attractors for the non-Newtonian fluid with delays under weaker assumptions (similar to that in [51]) with respect to the $t$-regularity.

Remark 6.4. Caraballo, Łukaszewicz and Real 13 proved the existence of a pullback attractor for a non-autonomous 2D Navier-Stokes model with a quite general nonautonomous term, a case in which the theory of uniform attractors does not work. We guess that the method in 13 could be extended to treat the Navier-Stokes model with delays and is also applicable to the non-Newtonian fluid with delays.

REMARK 6.5. Rosa 49] proved the existence of an $L^{2}$-compact global attractor for the Navier-Stokes equations on two-dimensional unbounded domains in which the Poincaré inequality holds. Later $\mathrm{Ju} 31$ extended the results of 49 by proving that the global attractor obtained in [49] is compact not only in the $L^{2}$-norm but also in the $H^{1}$-norm and first pointed out the regularity of the global attractor for the Naiver-Stokes equations without delays. We note that the pullback attractors of the retarded Navier-Stokes equations also bear the similar regularity.

Acknowledgments. We express our sincere thanks to the anonymous reviewers for their careful reading of the manuscript, correcting some English mistakes and typos. We also thank the editors for their kind help.

\section{REFERENCES}

[1] L. Arnold, "Random Dynamical Systems", Springer, Berlin, 1998. MR 1723992 (2000m:37087)

[2] R. A. Adams, "Sobolev Spaces," Academic Press, New York, 1975. MR0450957 (56:9247)

[3] A. V. Babin, M. I. Vishik, "Attractors of Evolution Equations", North-Holland, Amsterdam, 1992. MR.1156492 (93d:58090)

[4] Hyeong-Ohk Bae, Existence, regularity, and decay rate of solutions of non-Newtonian flow, J. Math. Appl. Anal., 231(1999), 467-491. MR1669171 (99m:35194)

[5] J. M. Ball, Continuity properties of global attractors of generalized semiflows and the Navier-Stokes equations, J. Nonlinear Sci., 7(1997), 475-502. MR1462276 (98j:58071a)

[6] H. Bellout, F. Bloom, J. Nečas, Weak and measure-valued solutions for non-Newtonian fluids, C. R. Acad. Sci. Paris, 317(1993), 795-800.

[7] H. Bellout, F. Bloom, J. Nečas, Young measure-valued solutions for non-Newtonian incompressible viscous fluids, Commun. PDE., 19(1994), 1763-1803. MR1301173 (95i:35227)

[8] F. Bloom, W. Hao, Regularization of a non-Newtonian system in an unbounded channel: Existence and uniqueness of solutions, Nonlinear Anal., 44(2001), 281-309. MR.1817094 (2002b:76003)

[9] F. Bloom, W. Hao, Regularization of a non-Newtonian system in an unbounded channel: Existence of a maximal compact attractor, Nonlinear Anal., 43(2001),743-766. MR1808208 (2001j:35026)

[10] M. Boukrouche, G. Łukaszewicz, J. Real, On pullback attractors for a class of two-dimensional turbulent shear flows, Internat. J. Eng. Sci., 44(2006), 830-844. MR2255762 (2008d:37157)

[11] H. Crauel, F. Flandoli, Attractors for random dynamical systems, Probab. Theory Related Fields, 100(1994), 365-393. MR1305587 (95k:58092)

[12] P. Constantin, C. Foias, "Navier Stokes Equations", The University of Chicago Press, Chicago, 1988. MR 972259 (90b:35190)

[13] T. Caraballo, G. Łukaszewicz, J. Real, Pullback attractors for non-autonomous 2D-Navier-Stokes equations in some unbounded domains, C. R. Math. Acad. Sci. Paris, Ser. I., 342(2006), 263-268. MR 2196010 (2006h:35201)

[14] T. Caraballo, G. Łukaszewicz, J. Real, Pullback attractors for asymptotically compact nonautonomous dynamical systems, Nonlinear Anal., 64(2006), 484-498. MR2191992 (2006h:37028) 
[15] T. Caraballo, J. A. Langa, Attractors for differential equations with variable delay, J. Math. Anal. Appl., 260(2001), 421-438. MR1845562 (2002f:37040)

[16] T. Caraballo, J. A. Langa, On the upper semicontinuity of cocycle attractors for non-autonomous and random dynamical systems, Dyn. Contin. Discrete Impulsive Syst. Ser. A Math. Anal. 10(2003), 491-513. MR 1978585 (2004c:37110)

[17] T. Caraballo, J. Real, Navier-Stokes equations with delays, Roy. Soc. London Proc. Ser. A Math. Phys. Eng. Sci., 457(2001), 2441-2453. MR1862662 (2002g:35168)

[18] T. Caraballo, J. Real, Asymptotic behaviour of Navier-Stokes equations with delays, Roy. Soc. London Proc. Ser. A Math. Phys. Eng. Sci., 459(2003),3181-3194. MR2027360 (2004k:35295)

[19] T. Caraballo, J. Real, Attractors for 2D-Navier-Stokes models with delays, J. Differential Equations, 205(2004), 271-297. MR2091818 (2005h:35266)

[20] T. Caraballo, P. E. Kloeden, P. Marin-Rubio, Weak pullback attractors of setvalued processes, J. Math. Anal. Appl., 288(2003), 692-707. MR2020190 (2004i:34025)

[21] T. Caraballo, P. Marin-Rubio, J. Valero, Autonomous and non-autonomous attractors for differential equations with delays, J. Differential Equations, 208(2005), 9-41. MR2107292 (2005i:37095)

[22] T. Caraballo, P. Marin-Rubio, J. Valero, Attractors for differential equations with unbounded delays, J. Differential Equations, 239(2007), 311-342. MR2344275 (2008g:34149)

[23] D. N. Cheban, P. E. Kloeden, B. Schmalfuss, The relationship between pullback, forwards and global attractors of nonautonomous dynamical systems, Nonlinear Dyn. Syst. Theory, 2(2002), 9-28. MR 1989935(2004e:34090)

[24] V. V. Chepyzhov, M. I. Vishik, "Attractors for Equations of Mathematical Physics", AMS Colloquium Publications, 49. Amer. Math. Soc., Providence, RI., 2002. MR.1868930(2003f:37001c)

[25] H. Crauel, A. Debussche, F. Flandoli, Random attractors, J. Dynam. Differential Equations, 9(1997), 307-341. MR.1451294 (98c:60066)

[26] F. Flandoli, B. Schmalfuss, Random attractors for the 3D stochastic Navier-Stokes equation with multiplicative white noise, Stochast. Stochast. Rep., 59(1996), 21-45. MR.1427258 (98g:60113)

[27] B. Guo, P. Zhu, Partial regularity of suitable weak solution to the system of the incompressible non-Newtonian fluids, J. Differential Equations, 178(2002), 281-297. MR 1879829 (2003a:35156)

[28] J. K. Hale, "Theory of Functional Differential Equations", Springer, Berlin, 1977. MR0508721 $(58: 22904)$

[29] J. K. Hale, "Asymptotic Behavior of Dissipative Systems", Amer. Math. Soc., Providence, R.I., 1988, MR941371 (89g:58059)

[30] J. K. Hale, S. M. Verduyn Lunel, "Introduction to Functional Differential Equations", SpringerVerlag, New York, 1993. MR 1243878(94m:34169)

[31] N. Ju, The $H^{1}$-compact global attractor for the solutions to the Navier-Stokes equations in 2D unbounded domains, Nonlinearity, 13(2000), 1227-1238. MR.1767956 (2001f:37131)

[32] N. Ju, Existence of global attractor for the three-dimensional modified Navier-Stokes equations, Nonlinearity, 14(2001), 777-786. MR1837637(2002d:76011)

[33] P. E. Kloeden, B. Schmalfuss, Nonautonomous systems, cocycle attractors and variable time-step discretization, Numer. Algorithms, 14(1997), 141-152. MR1456499(98f:58132)

[34] P. E. Kloeden, B. Schmalfuss, Asymptotic behaviour of nonautonomous difference inclusions, Syst. Cont. Lett., 33(1998), 275-280. MR1613094(99d:39006)

[35] Y. Kuang, "Delay Differential Equations with Applications in Population Dynamics", Academic Press, Boston, 1993. MR 1218880 (94f:34001)

[36] O. Ladyzhenskaya, New equations for the description of the viscous incompressible fluids and solvability in large of the boundary value problems for them, in "Boundary Value Problems of Mathematical Physics", Amer. Math. Soc., Providence, RI. 1970.

[37] O. Ladyzhenskaya, "Attractors for Semigroups and Evolution Equations", Cambridge University Press, Cambridge, 1991. MR1133627 (92k:58040)

[38] J. A. Langa, B. Schmalfuss, Finite dimensionality of attractors for non-autonomous dynamical systems given by partial differential equations, Stoch. Dyn., 4(2004), 385-404. MR2085975 (2005f:37169)

[39] J. A. Langa, G. Eukaszewicz, J. Real, Finite fractal dimension of pullback attractors for nonautonomous 2D Navier-Stokes equations in some unbounded domains, Nonlinear Anal., 66(2007), 735-749. MR:2274880 (2007h:37126)

[40] G. Łukaszewicz, W. Sadowski, Uniform attractor for 2D magneto-micropolar fluid flow in some unbounded domains, Zeitsch. Angew. Math. Phys., 55(2004), 247-257. MR2047286 (2005a:35031) 
[41] J. Málek, J. Nečas, M. Rokyta, M. Rúžičk, "Weak and Measure-valued Solutions to Evolutionary PDEs", Chapman and Hall, New York, 1996. MR.1409366 (97g:35002)

[42] M. Pokorný, Cauchy problem for the non-Newtonian viscous incompressible fluids, Appl. Math., 41(1996), 169-201. MR1382464 (97a:35190)

[43] J. Mallet-Paret, G. Sell, Systems of differential delay equations: Floquet multipliers and discrete Lyapunov functions, J. Differential Equations, 125(1996), 385-440. MR1378762 (97a:34193a)

[44] J. Mallet-Paret, G. R. Sell, The Poincaré-Bendixson theorem for monotone cyclic feedback systems with delay, J. Differential Equations, 125(1996), 441-489. MR.1378763 (97a:34193b)

[45] A. V. Rezounenko, J. Wu, A non-local PDE model for population dynamics with state-selective delay: Local theory and global attractors, J. Computational Appl. Math., 190(2006), 99-113. MR.2209496 (2006j:35233)

[46] A. V. Rezounenko, Partial differential equations with discrete and distributed state-dependent delays, J. Math. Anal. Appl., 326(2007), 1031-1045. MR2280961 (2008f:35396)

[47] J. C. Robinson, "Infinite-dimensional Dynamical Systems", Cambridge University Press, Cambridge, 2001. MR:1881888 (2003f:37001a)

[48] J. C. Robinson, A. Rodriguez-Bernal, A. Vidal-Lopez, Pullback attractors and extremal complete trajectories for non-autonomous reaction-diffusion problems, J. Differential Equations, 238(2007), 289-337. MR 2341427

[49] R. Rosa. The global attractor for the 2D Navier-Stokes flow on some unbounded domains, Nonlinear Anal., 32(1998), 71-85. MR1491614 (98k:35152)

[50] B. Schmalfuss, Backward cocycle and attractors of stochastic differential equations, in: V. Reitmann, T. Redrich, N.J. Kosch (Eds.), "International Seminar on Applied Mathematics-Nonlinear Dynamics: Attractor Approximation and Global Behaviour", 1992, pp. 185-192.

[51] B. Schmalfuss, Attractors for non-autonomous dynamical systems, in: "B. Fiedler, K. Gröger, J. Sprekels (Eds.), Proc. Equadiff.", 1999, Berlin, World Scientific, River Edge, NJ, 2000 684-689. MR:1870217 (2002h:37044)

[52] G. Sell, Y. You, "Dynamics of Evolutionary Equations", Springer, New York, 2002. MR.1873467 (2003f:37001b)

[53] R. Temam, "Infinite Dimensional Dynamical Systems in Mechanics and Physics", Springer, Berlin, 2nd ed., 1997. MR1441312(98b:58056)

[54] J. Wu, "Theory and Applications of Partial Functional Differential Equations", Applied Mathematical Sciences, 119, Springer-Verlag, New York, 1996. MR.1415838 (98a:35135)

[55] Y. Wang, C. Zhong, S. Zhou, Pullback attractors of nonautonomous dynamical systems, Discrete Contin. Dyn. Syst., 16(2006), 587-614. MR2257151 (2007f:37131)

[56] C. Zhao, Y. Li, $H^{2}$-compact attractor for a non-Newtonian system in two-dimensional unbounded domains, Nonlinear Anal., 7(2004), 1091-1103. MR2038738 (2004k:37177)

[57] C. Zhao, S. Zhou, $L^{2}$-compact uniform attractors for a nonautonomous incompressible non-Newtonian fluid with locally uniformly integrable external forces in distribution space, J. Math. Phys., 48(2007), 032702-1-032702-12. MR2314493 (2008f:37185)

[58] C. Zhao, S. Zhou, Y. Li, Trajectory attractor and global attractor for a 2D incompressible nonNewtonian fluid, J. Math. Anal. Appl., 325(2007), 1350-1362. MR2270089 (2007h:37130)

[59] C. Zhao, S. Zhou, Pullback attractors for a non-autonomous incompressible non-Newtonian fluid, J. Differential Equations, 238(2007), 394-425. MR2341431(2008f:37184) 